\title{
An ant navigation model based on Weber's law
}

\author{
Paulo Amorim ${ }^{1}$, Thierry Goudon ${ }^{2}$, Fernando Peruani ${ }^{3}$
}

\begin{abstract}
We analyze an ant navigation model based on Weber's law, where the ants move across a pheromone landscape sensing the area using two antennae. The key parameter of the model is the angle $2 \beta$ representing the span of the ant's sensing area. We show that when $\beta<\pi / 2$ ants are able to follow (straight) pheromone trails proving that for initial conditions close to the trail, there exists a Lyapunov function that ensures ant trajectories converge on and follow the pheromone trail, with these solutions being locally asymptotically stable. Furthermore, we indicate that the features of the ant trajectories such as convergence speed or oscillation wave length are controlled by the angle $\beta$. For $\beta>\pi / 2$, we present numerical evidence that indicates that ants are unable to follow pheromone trails. We also assess our model by comparing it to previous experimental results, showing that the solutions' behavior falls into biologically meaningful ranges. Our work provides solid mathematical support for experimental studies where it was found that ant perception follows a Weber's law, by proving that such models lead to the desired robust and stable trail following.
\end{abstract}

\section{Introduction}

The exact nature of how individual ants move on the trail and respond to pheromone remains unknown. [24, p.1]

The study of how organized collective animal behavior arises from simple individual rules has been a fertile field of study for many years, see reference [31] for a detailed introduction to the topic. Ant societies, in particular, have been a focus of such investigations, due to their abundance in the biosphere, the relative ease with which they can be analyzed in the laboratory, and because the highly complex, seemingly intelligent behaviors observed in ant colonies are unsurpassed in the natural world.

One of the key aspects that allows the emergence of collective behavior in ant societies is chemical communication by way of pheromones. While pheromones

\footnotetext{
${ }^{1}$ Corresponding author. Instituto de Matemática, Universidade Federal do Rio de Janeiro, Av. Athos da Silveira Ramos 149, Centro de Tecnologia - Bloco C, Cidade Universitária - Ilha do Fundão, Caixa Postal 68530, 21941-909 Rio de Janeiro, RJ - Brasil

Email: paulo@im.ufrj.br. web page: http://www.im.ufrj.br/paulo/

${ }^{2}$ Université Côte d'Azur, Inria, CNRS, LJAD Email: thierry.goudon@inria.fr

${ }^{2}$ Université Côte d'Azur, CNRS, LJAD Email: fernando.peruani@unice.fr
} 
are used in virtually every aspect of ant life, they are crucial to ant foraging, especially when it relies on pheromone trails. Ants use pheromone trails to ultimately communicate between themselves information about food sources, such as direction, distance, quality, abundance, and so on [20, 33, 34.

Therefore, numerous experimental works have been dedicated to studying and modeling how ants respond to pheromone at the individual level. Here, we name just a few from a vast literature [1, 3, 15, 6, 11, 13, 14, 19, 21, 22, 23, 25, 26, 29, 27, 32] and concentrate on the important contributions in [4, 17, 16, 24, 27, 30]. In [4], an individual-based model (IBM) is proposed, using directed pheromones to mediate the interaction between ants. In [17, a kinetic-type PDE model is developed. In [27, an IBM model is presented, where formation of lanes is exhibited. Finally, in [16, 24, valuable experimental results are reported, and corresponding individual-based models are studied. In particular, the authors show experimentally that individual ants' turning rate is governed by Weber's law: the turning rate is determined by the difference in pheromone on both sides of the ant, divided by the sum of pheromone on each side. As we shall see, an appropriate formulation of Weber's law is a cornerstone of our analysis.

However, until now, to the best of our knowledge (and despite advanced attempts such as [16, 24, 27]), the rigorous mathematical study of such individual models is very scarce: for instance, stability results ensuring that trail-following behavior is robust with respect to small perturbations are still lacking. Thus the main goals in this paper are:

1. To present an individual-based model of ant navigation derived from modeling first principles using a generalization of Weber's law;

2. To provide a rigorous mathematical analysis of the stability properties of the model; and,

3. To provide analytical and numerical evidence indicating that a sensing area half-angle $\beta$ less that $\pi / 2$ is a necessary and sufficient condition for robust and stable trail following behavior.

To achieve these goals, we present an individual-based model (and variants of it) that is closely related to the models proposed in [16, 24, 2]. The proposed model presents a series of improvements to these pioneering works in term of their mathematical formulation. We provide a concise modeling framework, relying on very straightforward assumptions about ant movement and reaction to pheromone, and arrive at a general version of Weber's law. At the same time, we show rigorous mathematical results showing that, under certain realistic conditions, and especially when the sensing half-angle $\beta$ is less than $\pi / 2$, individuals indeed follow pheromone trails.

\subsection{Main results}

Our main contribution is to show that the proposed model provides an accurate and robust description of ant navigation, leading to trail following behavior. 
This is achieved through rigorous stability results for the differential equations governing the models.

One prominent feature of our model is the sensing area around the ant, on which it can sense pheromone. It is a circular sector with radius $\ell$ and sensing half-angle $\beta$ (so that the circular sector spans an angle $2 \beta$ ), illustrated in Figure 1. One question that arises naturally is to explain the observation that ant antennae seem to be, in all cases, oriented towards the front of the ant [24, 20]. Although this seems intuitively to be the best possible arrangement, it is not entirely clear that another configuration, also spanning regions behind or to the sides of the ant could not provide an advantage for pheromone detection.

It turns out that our model supports the empirical observation that the sensing half-angle $\beta$ should be less than $\pi / 2$. Indeed, many of our mathematical results related to trail-following hold under the assumption that $\beta<\pi / 2$; this means that the ant does not detect pheromone behind it.

To be more precise, the main mathematical results of this paper are the stability results in Section 3. We prove that under the assumption that the sensing half-angle $\beta$ is less than $\pi / 2$, trail following is stable. This means that the solution of the individual-based model that perfectly follows the middle of a pheromone trail is linearly stable, under reasonable conditions on the pheromone concentration defining the trail. Furthermore, we prove the existence of a Lyapunov function, thus ensuring the asymptotic stability of the trajectories.

Even before establishing the above-mentioned stability results, another, more basic property of our model is ensured only when $\beta<\pi / 2$ : that is a lower bound on the velocity of the ant. Although in nature ants do stop occasionally, it has been observed that when following a trail, the speed of each individual remains fairly constant 24. In fact, the formulation we choose does not allow zero velocity, since the sensing region is defined around the ant's velocity vector using its direction. It turns out that when $\beta<\pi / 2$ the ant's velocity will always tend to approach a range of "natural" velocities which are bounded and bounded away from zero. In contrast, when $\beta>\pi / 2$ there is no such range of velocities.

\subsection{Outline of the paper}

In Section 2, we present our general model based on Weber's law and the variants we study. In Section 3, we state and prove our rigorous stability and wellposedness results. Next, in Section 4 we discuss the role of the sensing area angle parameter on the trail following ability of individuals. We continue with an assessment of the model using a comparison with some experimental results in Section 5, before stating some concluding remarks and outlook in the final section.

\section{Presentation of the model}

We propose an individual based model of ant foraging based on the simple assumption that ants are attracted to the presence of pheromone on a small 


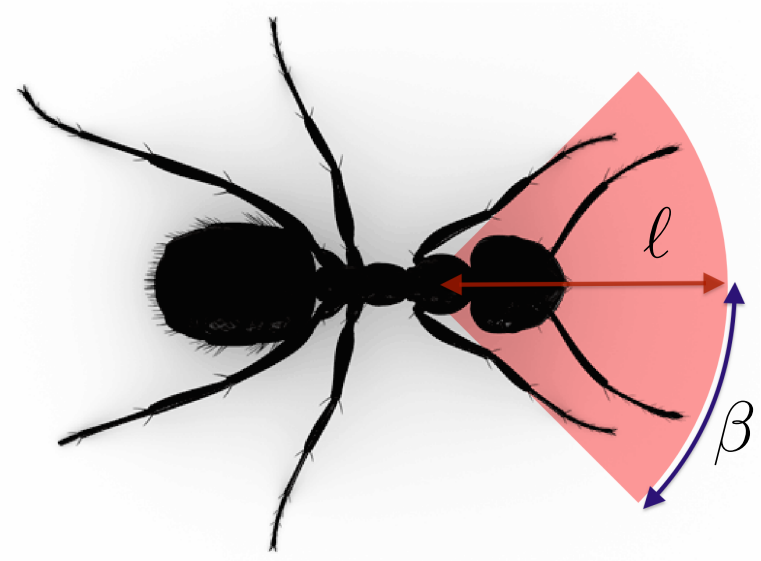

Figure 1: Illustration of the sensing area $B$ around an ant, defined by the halfangle $\beta$ and radius $\ell$.

sensing area determined by the direction the ant is facing, modeling the reach of the antennae. The center of the sensing area is assumed to be the pivotal point on the ant as it changes direction. A similar assumption is implicit in the modeling strategies of [16, 24]. However, here we work from first principles in a more concise way to derive the differential equations modeling the ant's behavior. The consequence is that we are able to not only obtain rigorous mathematical results, but also to analyze the role of the parameters in the dynamics, most importantly the angle of the sensing area.

\subsection{Individual dynamics and a generalized Weber's law}

In general form, our model is of the type

$$
\left\{\begin{array}{l}
\dot{\mathbf{x}}(t)=\mathbf{v}(t), \\
\dot{\mathbf{v}}(t)=-\frac{1}{\tau}(\mathbf{v}-F(\mathbf{x}, \mathbf{v})),
\end{array}\right.
$$

where $\mathbf{x}(t)=\left(\mathbf{x}_{1}(t), \mathbf{x}_{2}(t)\right)$ is the position of the ant, $\mathbf{v}(t)=\left(\mathbf{v}_{1}(t), \mathbf{v}_{2}(t)\right)$ is the velocity, and the desired velocity $F(\mathbf{x}, \mathbf{v})$ must be specified. The system 2.1) describes Newton's second law with dissipation (the term proportional to $\mathbf{v}$ ), where the change in velocity is given by a relaxation to the desired velocity $F$, with (small) relaxation time $\tau$.

The main assumption of our model is that ants are attracted to pheromone present in a small sensing area around them, illustrated in Figure 1. This sensing area is a circular sector aligned with the direction the ant is facing, and represents the area around the ant's head where it is able to detect pheromone by antennation. At each instant, the ant makes an average of the points on the 
circular sector, weighted by quantity of pheromone at each point. This average point can be seen as a vector from the position of the ant to the direction in which the average pheromone is greatest - which will not necessarily be the direction of the gradient of pheromone concentration.

More precisely, let $\ell$ be the radius of the sensing area, $\angle\left(\mathbf{v}, \mathbf{v}^{\prime}\right) \in[-\pi, \pi]$ the angle from the vector $\mathbf{v}$ to the vector $\mathbf{v}^{\prime}$, and let $\beta$ be half the angle of the sensing area $B(\mathbf{v}, \ell, \beta)$, which is a circular sector of radius $\ell$ centered at zero and aligned with the direction of the velocity vector $\mathbf{v}$ (see Figure 1):

$$
B(\mathbf{v}, \ell, \beta)=\left\{\mathbf{y} \in \mathbb{R}^{2}: \angle(\mathbf{v}, \mathbf{y}) \in(-\beta, \beta),\|\mathbf{y}\| \leq \ell\right\} .
$$

Introduce now a given, non-negative pheromone concentration $\mathcal{P}(t, \mathbf{x})$. We set the desired velocity $F(\mathbf{x}, \mathbf{v})$ as the vector

$$
F(\mathbf{x}, \mathbf{v})=\lambda \int_{B} \mathbf{y} \frac{\mathcal{P}(t, \mathbf{x}+\mathbf{y})}{\int_{B} \mathcal{P}(t, \mathbf{x}+\mathbf{y}) d \mathbf{y}} d \mathbf{y}
$$

where $B=B(\mathbf{v}, \ell, \beta)$ is defined in $(2.2)$ above, and $\lambda$ is a constant with units $(\text { time })^{-1}$. The normalization by

$$
\int_{B} \mathcal{P}(t, \mathbf{x}+\mathbf{y}) d \mathbf{y}
$$

corresponds to the total stimuli normalization in Weber's law 2.4 - see the discussion below. Note that the numerator in 2.3 is a vector, and we abuse the notation by writing the two-dimensional Lebesgue measure as $d \mathbf{y}$.

\subsubsection{Relation to Weber's law}

In the study of Ecology, animal movement, and, more generally, in situations where an organism must respond to stimuli from different sources or directions, whose intensity may span orders of magnitude, the so-called Weber's law applies. It states, roughly, that if $L$ is stimulus detection on the left side, and $R$ on the right side, then

$$
\text { Individual response } \simeq \frac{L-R}{L+R}
$$

In 24], Weber's law as applied to ant movement was shown to hold experimentally. The authors of that work argue convincingly about why such a result apparently contradicts earlier experiments which appeared to show that a different, nonlinear (in $L$ and $R$ ) response law holds for ant movement (see [28] and the discussion in 24]). Also, the model in [17] can be seen as a version of Weber's law. Here, we support and extend the conclusions in [24] related to the applicability of Weber's law to ant movement. Indeed, our model (2.3) may be seen as a generalized Weber's law: both the $L-R$ term in $(2.4)$ and the outermost integral in 2.3 represent an average of directional stimulus, in which signals from opposing directions have a canceling effect. In turn, the normalization $L+R$ in (2.4) corresponds to the denominator in (2.3). 


\subsection{Effective pheromone concentration}

The model (2.1) with desired velocity 2.3 has the drawback of not being clearly defined when the pheromone concentration tends to zero in a spatially non homogeneous way. It is not easy to see how 2.3 should be interpreted when pheromone levels are very low or zero. However, suppose that the pheromone concentration is a constant in space in a region around the ant. Then, since the sensing area $B$ is symmetric with respect to the velocity vector $\mathbf{v}$, the desired velocity in 2.3 simplifies to

$$
F(\mathbf{x}, \mathbf{v})=\frac{\lambda}{|B|} \int_{B} \mathbf{y} d \mathbf{y}=C \frac{\mathbf{v}}{\|\mathbf{v}\|},
$$

where $C=\lambda \frac{2}{3} \ell \frac{\sin \beta}{\beta}$ depends only on the physical parameters of the problem, but not on the pheromone concentration (see 2.15, 2.14 below for the calculation of $C$ ). We propose that this "natural velocity" should also be valid when the pheromone concentration is zero.

Now, it is well known 20] that there is a minimum pheromone concentration threshold below which individuals either ignore or cannot detect pheromone. In turn, in the absence of pheromone, ants typically perform a random search [16, 24, 20]. Therefore it would be desirable if our model exhibits continuity of behavior between a state where pheromone is absent - and the ant is governed by the natural velocity 2.5 - and a state with just-noticeable pheromone.

To achieve this continuity property, we propose to replace the pheromone concentration $\mathcal{P}(t, \mathbf{x})$ appearing in 2.3 with a suitable function $\mathcal{P}_{e}(t, \mathbf{x})$ which we call the effective pheromone. This function should take into account the fact that, in the limit as the pheromone concentration reaches below a certain threshold, the ant's behavior should approach the behavior determined by $(2.5)$.

Thus, we introduce the minimum pheromone threshold $c_{*}>0$ as the minimum amount of pheromone which an individual reacts to. Moreover, since there is only a finite amount of chemical receptors in an ant's antennae, it is natural to assume that ants are not sensitive to pheromone concentrations above a certain maximum saturation concentration $c^{*}$. Therefore, we define the effective pheromone as

$$
\mathcal{P}_{e}(t, \mathbf{x})=\min \left(c^{*}, \max \left(c_{*}, \mathcal{P}(t, \mathbf{x})\right)\right) .
$$

Then, the effective desired velocity reads

$$
F(\mathbf{x}, \mathbf{v})=\lambda \int_{B} \mathbf{y} \frac{\mathcal{P}_{e}(t, \mathbf{x}+\mathbf{y})}{\int_{B} \mathcal{P}_{e}(t, \mathbf{x}+\mathbf{y}) d \mathbf{y}} d \mathbf{y}
$$

where $\mathcal{P}_{e}$ is given by $(2.6)$ with $\mathcal{P}$ the (actual) physical concentration of pheromone. We will see in Section 3 below how using the effective pheromone concentration $\mathcal{P}_{e}$ will be essential to obtain our stability results.

Remark 2.1. $\quad$ 1. Any function $\mathcal{P}_{e}(t, \mathbf{x})$ verifying for all $t>0, \mathbf{x} \in \mathbb{R}^{2}$

$$
0<c_{*} \leq \mathcal{P}_{e}(t, \mathbf{x}) \leq c^{*}
$$


can be considered an effective pheromone concentration, without need for an underlying physical pheromone concentration. Therefore, in the remainder of this paper, when we refer to an effective pheromone, we will mean a function satisfying (2.7).

2. Although it is is not used in the analysis, it seems relevant to assume that the effective pheromone concentration $\mathcal{P}_{e}$ is a non decreasing function of the actual concentration $\mathcal{P}$.

3. Other choices for 2.8 are possible; namely, one could choose a sigmoid function connecting $c_{*}$ when $\mathcal{P}$ is zero with $c^{*}$ when $\mathcal{P}$ goes to $+\infty$. We work with 2.8 for definiteness only, since the results of this paper can be easily adapted to different choices of effective pheromone concentration.

\subsection{Generalizing the model with a local sensing measure}

In the expression for the desired velocity in (2.7), we assume that every point on the sensing area $B$ gives an equal contribution to the integral. While this may serve as a first approximation, in practice ants sense pheromone with the tips of their antennae, which touch the substrate only in a subregion of the sensing area $B$ [24]. Different species of ants move their antennae in different ways, some maintaining a rigid configuration of the antennae with respect to the ant's body, while others move the antennae quickly around their sensing area [15].

In order to include these effects in our model, we introduce a nonnegative sensing measure $\mu \in \mathcal{M}(B)$ in the following natural way. Choose an arbitrary unit vector $\overline{\mathbf{v}}$, and consider a positive measure $\bar{\mu}$ supported in a circular sector around $\overline{\mathbf{v}}$. Suppose that $\bar{\mu}$ is symmetric with respect to $\overline{\mathbf{v}}$. Then, we define $\beta$ and $\ell$ from $\bar{\mu}$ as the smallest values for which the set $B(\overline{\mathbf{v}}, \ell, \beta)$ defined in 2.2 contains the support of $\bar{\mu}$. Then, for any vector $\mathbf{v}$, simply define the measure $\mu$ on $B$ by applying the same rotation that transforms $\overline{\mathbf{v}}$ into $\mathbf{v} /\|\mathbf{v}\|$.

Then the desired velocity 2.7 reads

$$
F_{\mu}(\mathbf{x}, \mathbf{v})=\lambda \int_{B} \mathbf{y} \frac{\mathcal{P}_{e}(t, \mathbf{x}+\mathbf{y})}{\int_{B} \mathcal{P}_{e}(t, \mathbf{x}+\mathbf{y}) d \mu(\mathbf{y})} d \mu(\mathbf{y})
$$

When the measure $\mu$ can be given by some integrable function $\mu: B \rightarrow \mathbb{R}$ we write

$$
F_{\mu}(\mathbf{x}, \mathbf{v})=\lambda \int_{B} \mathbf{y} \frac{\mu(\mathbf{y}) \mathcal{P}_{e}(t, \mathbf{x}+\mathbf{y})}{\int_{B} \mu(\mathbf{y}) \mathcal{P}_{e}(t, \mathbf{x}+\mathbf{y}) d \mathbf{y}} d \mathbf{y}
$$

In the particular case where $\mu \equiv 1$, then $F_{\mu}$ reduces to $F$ given by 2.7 . This models a case where antennal movement is important, and so every point on the

sensing area detects pheromone with equal sensitivity. In contrast, if $\mu$ contains Dirac delta measures, then the ant's antennae only touch the substrate at some isolated points. 


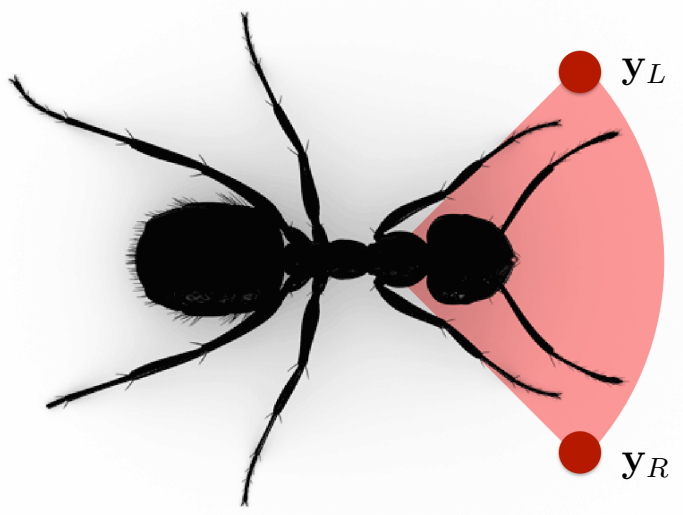

Figure 2: Sensing points for the two-point sensing model 2.11.

\subsection{A model with two-point sensing}

Let us consider in more detail the case where

$$
\mu=\delta_{\mathbf{y}=\mathbf{y}_{L}}+\delta_{\mathbf{y}=\mathbf{y}_{R}} .
$$

Here, $\delta$ is the Dirac delta and $\mathbf{y}_{L}\left(\right.$ resp. $\left.\mathbf{y}_{R}\right)$ represent the points where the tip of the left (right) antenna touches the substrate, at fixed angles $\pm \beta$ from the ant's direction, at a distance of $\ell$. Thus $\mathbf{y}_{L}$ and $\mathbf{y}_{R}$ are at the two opposite "corners" of $B$ from the position of the ant (see Figure 2):

$$
\mathbf{y}_{L}=\ell\left(\begin{array}{c}
\cos (\Theta+\beta) \\
\sin (\Theta+\beta)
\end{array}\right), \quad \mathbf{y}_{R}=\ell\left(\begin{array}{c}
\cos (\Theta-\beta) \\
\sin (\Theta-\beta)
\end{array}\right),
$$

where $\mathbf{v}=\|\mathbf{v}\|(\cos \Theta, \sin \Theta)$. This models the case, in contrast to $\mu \equiv 1$, where the antennae have little or no mobility relative to the ant's head.

Assuming 2.11, 2.12, the desired velocity (2.9) has the expression:

$$
F(\mathbf{x}, \mathbf{v})=\frac{\mathbf{y}_{L} \mathcal{P}_{e}\left(t, \mathbf{x}+\mathbf{y}_{L}\right)+\mathbf{y}_{R} \mathcal{P}_{e}\left(t, \mathbf{x}+\mathbf{y}_{R}\right)}{\mathcal{P}_{e}\left(t, \mathbf{x}+\mathbf{y}_{L}\right)+\mathcal{P}_{e}\left(t, \mathbf{x}+\mathbf{y}_{R}\right)}
$$

\subsection{A linearized model}

In this section, we deduce from (2.3) a new model in which the pheromone concentration $\mathcal{P}(t, \mathbf{x}+\mathbf{y})$ appearing in 2.3 is linearized around the spatial point $\mathbf{x}$. This linearized model has the advantage that the character of the spatial anisotropy introduced by the directional bias in the ant's perception is clearly revealed, by the presence of a nematic tensor. However, it is only suitable for cases where the radius of the sensing area is very small with respect 
to some characteristic measure of the physical situation considered. Still, it may be a useful formulation having in mind the derivation of meso- or macroscopic models from the proposed individual-based model.

Making a Taylor development of $\mathcal{P}$, in space, around $(t, \mathbf{x})$, we find

$$
\mathcal{P}(t, \mathbf{x}+\mathbf{y}) \simeq \mathcal{P}(t, \mathbf{x})+\mathbf{y} \cdot \nabla \mathcal{P}(t, \mathbf{x})
$$

We linearize the numerator of 2.3 accordingly:

$$
\begin{aligned}
\int_{B} \mathbf{y} \mathcal{P}(t, \mathbf{x}+\mathbf{y}) d \mathbf{y} & \simeq \int_{B} \mathbf{y} \mathcal{P}(t, \mathbf{x}) d \mathbf{y}+\int_{B} \mathbf{y} \otimes \mathbf{y} \nabla \mathcal{P}(t, \mathbf{x}) d \mathbf{y} \\
& =\mathcal{P}(t, \mathbf{x}) \int_{B} \mathbf{y} d \mathbf{y}+\int_{B} \mathbf{y} \otimes \mathbf{y} d \mathbf{y} \nabla \mathcal{P}(t, \mathbf{x})
\end{aligned}
$$

For the denominator of 2.3 we find

$$
\begin{aligned}
\int_{B} \mathcal{P}(t, \mathbf{x}+\mathbf{y}) d \mathbf{y} & \simeq \int_{B} \mathcal{P}(t, \mathbf{x}) d \mathbf{y}+\int_{B} \mathbf{y} \nabla \mathcal{P}(t, \mathbf{x}) d \mathbf{y} \\
& =\mathcal{P}(t, \mathbf{x})|B|+\nabla \mathcal{P}(t, \mathbf{x}) \cdot \int_{B} \mathbf{y} d \mathbf{y}
\end{aligned}
$$

We write the velocity vector as $\mathbf{v}=\|\mathbf{v}\|(\cos \Theta, \sin \Theta)$. Then some lengthy but straightforward calculation gives

$$
\begin{gathered}
|B(\mathbf{v}, \ell, \beta)|=\beta \ell^{2} \\
\int_{B} \mathbf{y} d \mathbf{y}=\ell^{3} \frac{2}{3} \sin \beta(\cos \Theta, \sin \Theta)
\end{gathered}
$$

and

$$
\int_{B} \mathbf{y} \otimes \mathbf{y} d \mathbf{y}=\frac{\ell^{4}}{4}\left(\beta \mathbf{I}+\frac{1}{2} \sin 2 \beta A(\Theta)\right),
$$

with the so-called nematic tensor $A$ given by

$$
\begin{aligned}
A(\Theta) & =\left(\begin{array}{lr}
\cos ^{2} \Theta-\sin ^{2} \Theta & 2 \cos \Theta \sin \Theta \\
2 \cos \Theta \sin \Theta & -\cos ^{2} \Theta+\sin ^{2} \Theta
\end{array}\right) \\
& =\left(\begin{array}{lr}
\cos 2 \Theta & \sin 2 \Theta \\
\sin 2 \Theta & -\cos 2 \Theta
\end{array}\right) .
\end{aligned}
$$

Then the linearized version of the desired velocity 2.3 reads (recall that the 
sensing area $B$ is given in $(2.2)$

$$
\begin{aligned}
& F_{\operatorname{lin}}(\mathbf{x}, \mathbf{v})=\lambda \frac{\mathcal{P}(t, \mathbf{x}) \int_{B} \mathbf{y} d \mathbf{y}+\int_{B} \mathbf{y} \otimes \mathbf{y} d \mathbf{y} \nabla \mathcal{P}(t, \mathbf{x})}{\mathcal{P}(t, \mathbf{x})|B|+\nabla \mathcal{P}(t, \mathbf{x}) \cdot \int_{B} \mathbf{y} d \mathbf{y}} \\
& =\lambda \frac{\mathcal{P}(t, \mathbf{x}) \ell \frac{2}{3} \sin \beta(\cos \Theta, \sin \Theta)+\frac{\ell^{2}}{4}\left(\beta \mathbf{I}+\frac{1}{2} \sin 2 \beta A(\Theta)\right) \nabla \mathcal{P}(t, \mathbf{x})}{\mathcal{P}(t, \mathbf{x}) \beta+\nabla \mathcal{P}(t, \mathbf{x}) \cdot \ell \frac{2}{3} \sin \beta(\cos \Theta, \sin \Theta)} .
\end{aligned}
$$

Note that the matrix $A$ is the reflection on the line with direction $\mathbf{v}$, as can be checked by noting that $\operatorname{det} A=-1$ and for any vector $\mathbf{u}$, we have $\mathbf{v} \cdot A \mathbf{u}=\mathbf{v} \cdot \mathbf{u}$. This, in conjunction with 2.16), yields that the linearized desired velocity $F_{\text {lin }}$ is parallel to the traveling direction $\mathbf{v}$, in the case where $\nabla \mathcal{P}$ is parallel to $\mathbf{v}$.

We can go a step further with the approximation

$$
\begin{aligned}
\tilde{F}_{\operatorname{lin}}(\mathbf{x}, \mathbf{v}) & =\lambda \frac{\int_{B} \mathbf{y} d \mathbf{y}}{|B|}+\lambda\left(\frac{\int_{B} \mathbf{y} \otimes \mathbf{y} d \mathbf{y}}{|B|}-\frac{\int_{B} \mathbf{y} d \mathbf{y} \otimes \int_{B} \mathbf{y} d \mathbf{y}}{|B|^{2}}\right) \frac{\nabla \mathcal{P}(t, \mathbf{x})}{\mathcal{P}(t, \mathbf{x})} \\
& =\lambda \ell \frac{2}{3} \frac{\sin \beta}{\beta}(\cos \Theta, \sin \Theta)+\lambda \ell^{2} \tilde{A}(\Theta) \frac{\nabla \mathcal{P}(t, \mathbf{x})}{\mathcal{P}(t, \mathbf{x})} .
\end{aligned}
$$

The Cauchy-Schwarz inequality tells us that the matrix

$$
\ell^{2} \tilde{A}(\Theta)=\int_{B} \mathbf{y} \otimes \mathbf{y} \frac{d \mathbf{y}}{|B|}-\int_{B} \mathbf{y} \frac{d \mathbf{y}}{|B|} \otimes \int_{B} \mathbf{y} \frac{d \mathbf{y}}{|B|}
$$

is symmetric and definite-positive (for the continuous model 2.37; for the twopoints measure (2.13), the kernel would be spanned by $\mathbf{v}$ ). We have

$$
\begin{aligned}
\tilde{A}(\Theta) & =\frac{1}{4}\left(\mathbf{I}+\frac{\sin (2 \beta)}{2 \beta} A(\Theta)\right)-\frac{4}{9} \frac{\sin ^{2}(\beta)}{\beta^{2}}\left(\begin{array}{cr}
\cos ^{2} \Theta & \cos \Theta \sin \Theta \\
\cos \Theta \sin \Theta & \sin ^{2} \Theta
\end{array}\right) \\
& =\frac{1}{4}\left(\mathbf{I}+\frac{\sin (2 \beta)}{2 \beta} A(\Theta)\right)-\frac{2}{9} \frac{\sin ^{2}(\beta)}{\beta^{2}}(\mathbf{I}+A(\Theta)) \\
& =\frac{1}{4}\left(1-\frac{8 \sin ^{2}(\beta)}{9 \beta^{2}}\right) \mathbf{I}+\frac{1}{4}\left(\frac{\sin (2 \beta)}{2 \beta}-\frac{8 \sin ^{2}(\beta)}{9 \beta^{2}}\right) A(\Theta) .
\end{aligned}
$$

Again, we readily check that $F_{\text {lin }}$ is aligned to the traveling direction $\mathbf{v}$, when $\nabla \mathcal{P}$ is proportional to $\mathbf{v}$. 


\section{Well-posedness and stability results}

In this section, we prove several mathematical results for the models presented previously. First, we establish non-degeneracy of the general model $[2.1,, 2.10)$ in the sense that the velocity vector remains bounded away from zero if, and only if, the sensing half-angle $\beta$ is less than $\pi / 2$. These a priori estimates then allows us to prove a well-posedness result.

Next, we show that for a given pheromone concentration representing a trail, the ant trajectory which follows the crest of the trail is linearly stable, both for models 2.7) and 2.13). The result can be strengthened when it is assumed that $\beta<\pi / 2$.

\subsection{Lower and upper bounds for the velocity}

The normalisation (2.7), 2.8 allows us to prove that in fact the magnitude of the ant's velocity vector remains bounded, and bounded away from zero, in the case where the sensing half-angle $\beta$ is less than $\pi / 2$. This property of the model is essential, especially the fact that the velocity vector is bounded away from zero. Indeed, the model breaks down if $\mathbf{v}=0$, since in that case the sensing area $B$ is not defined.

Proposition 3.1. Suppose that the sensing half-angle $\beta$ satisfies $0<\beta<\pi / 2$. Let $0<c_{*}<c^{*}$ be the pheromone detection thresholds in (2.8), and let $\mathcal{P}_{e}$ be any effective pheromone concentration (cf. (2.7)). Then, there exist constants $C_{1}, C_{2}>0$, depending only on $\ell, c_{*}, c^{*}, \beta$ and the initial data, such that for all $t \geq 0$

$$
\min \left(|\mathbf{v}(0)|, C_{1}\right) \leq|\mathbf{v}(t)| \leq \max \left(|\mathbf{v}(0)|, C_{2}\right),
$$

where $\mathbf{v}(t)$ is a solution to 2.1, 2.7. Moreover,

$$
C_{1}=\frac{2}{3} \lambda \ell \frac{c_{*} \sin \beta}{c^{*} \beta}, \quad C_{2}=\lambda \ell
$$

Proof. First, note that from the definition of $B$ in 2.2 we immediately obtain the bound

$$
|F(\mathbf{x}, \mathbf{v})| \leq \lambda \int_{B}|\mathbf{y}| \frac{\mathcal{P}_{e}(t, \mathbf{x}+\mathbf{y})}{\int_{B} \mathcal{P}_{e}(t, \mathbf{x}+\mathbf{y}) d \mathbf{y}} d \mathbf{y} \leq \lambda \ell \int_{B} \frac{\mathcal{P}_{e}(t, \mathbf{x}+\mathbf{y})}{\int_{B} \mathcal{P}_{e}(t, \mathbf{x}+\mathbf{y}) d \mathbf{y}} d \mathbf{y}=\lambda \ell
$$

and this is valid either for 2.7 or 2.3 .

Next, from the second equation of (2.1), we get

$$
\frac{1}{2} \frac{d}{d t}|\mathbf{v}|^{2}=-\frac{1}{\tau}|\mathbf{v}|^{2}+\frac{1}{\tau} F \cdot \mathbf{v}
$$

Using Cauchy-Schwartz inequality and the bound 3.2 , we find

$$
\frac{d}{d t}|\mathbf{v}|^{2} \leq-\frac{1}{\tau}|\mathbf{v}|^{2}+\frac{1}{\tau}|F|^{2} \leq-\frac{1}{\tau}|\mathbf{v}|^{2}+\frac{1}{\tau}(\lambda \ell)^{2}
$$


A simple comparison argument then shows that in fact $\mathrm{t}^{1}$

$$
|\mathbf{v}(t)| \leq \max (|\mathbf{v}(0)|, \lambda \ell), \quad t \geq 0
$$

which proves the upper bound in 3.1).

Returning to (3.3), let us assume for the moment that we know that

$$
F \cdot \mathbf{v} \geq C|\mathbf{v}|
$$

for some $C>0$. Then,

$$
\frac{1}{2} \frac{d}{d t}|\mathbf{v}|^{2} \geq-\frac{1}{\tau}|\mathbf{v}|^{2}+\frac{C}{\tau}|\mathbf{v}|
$$

and so

$$
|\mathbf{v}(t)| \geq \min (|\mathbf{v}(0)|, C), \quad t \geq 0,
$$

which is the remaining inequality in 3.1). It remains to prove (3.4). Write 2.7 as

$$
\lambda \int_{B} \mathcal{P}_{e}(t, \mathbf{x}+\mathbf{y}) d \mathbf{y} F(\mathbf{x}, \mathbf{v})=\int_{B} \mathbf{y} \mathcal{P}_{e}(t, \mathbf{x}+\mathbf{y}) d \mathbf{y}
$$

and take the scalar product with $\mathbf{v}$ on both sides. Using 2.15), we obtain for the right-hand side

$$
\begin{aligned}
\lambda \mathbf{v} \cdot \int_{B} \mathbf{y} \mathcal{P}_{e}(t, \mathbf{x}+\mathbf{y}) d \mathbf{y}= & \lambda c_{*} \mathbf{v} \cdot \int_{B} \mathbf{y} d \mathbf{y} \\
& +\lambda \int_{B} \mathbf{v} \cdot \mathbf{y}\left(\mathcal{P}_{e}(t, \mathbf{x}+\mathbf{y})-c_{*}\right) d \mathbf{y} \\
\geq & \lambda c_{*} \ell^{3} \frac{2}{3} \sin \beta|\mathbf{v}|,
\end{aligned}
$$

since, by 2.8), the function $\mathcal{P}_{e}(t, \mathbf{x}+\mathbf{y})-c_{*}$ is nonnegative and, due to the assumption $\beta<\pi / 2$, we have $\mathbf{v} \cdot \mathbf{y} \geq 0$. Next, we have by (2.14)

$$
\int_{B} \mathcal{P}_{e}(t, \mathbf{x}+\mathbf{y}) d \mathbf{y} \leq|B| c^{*}=\beta \ell^{2} c^{*} .
$$

Putting the previous estimates together, we find

$$
F \cdot \mathbf{v} \geq \frac{2}{3} \lambda \ell \frac{c_{*} \sin \beta}{c^{*} \beta}|\mathbf{v}|,
$$

which proves (3.4). This concludes the proof of the proposition.

In particular, the result of Proposition 3.1 tells us that if we take $|\mathbf{v}(0)|$ small enough, then the speed $|\mathbf{v}(t)|$ cannot decrease for small times. The case is different when the sensing area is larger than a half-circle, as the following result shows.

\footnotetext{
${ }^{1}$ When $|\mathbf{v}|$ is greater than $\lambda \ell,|\mathbf{v}|$ decreases; when it is smaller, it increases.
} 
Proposition 3.2. Suppose that the sensing half-angle satisfies $\beta>\pi / 2$. Then, for any initial data, there exist pheromone detection thresholds $0<c_{*}<c^{*}$ and effective pheromone concentrations such that $\frac{d}{d t}|\mathbf{v}(t)|_{\mid t=0}<0$.

Proof. With $\beta>\pi / 2$, define the angle $\theta \in(0, \pi / 2)$ by $\beta=\pi / 2+\theta$, as in Figure 3.

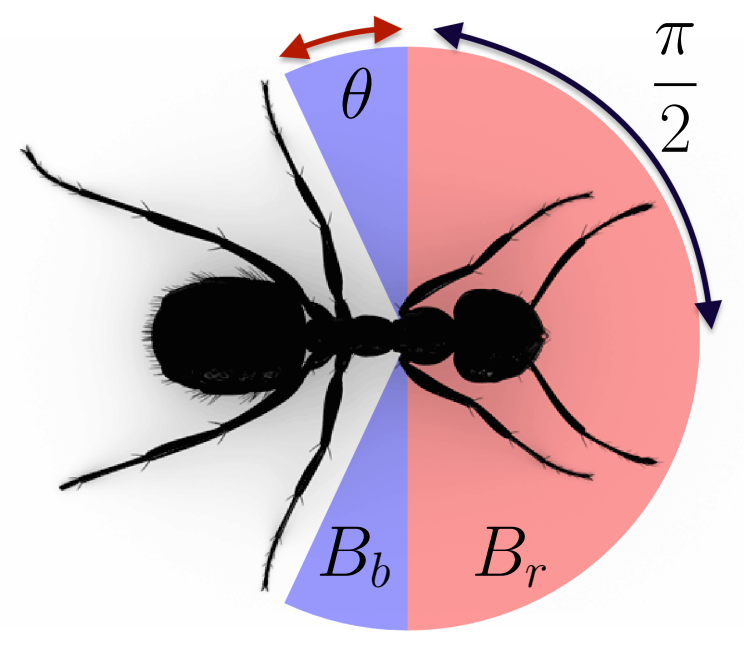

Figure 3: The sensing area $B$ larger than a half-circle.

Suppose that the pheromone has concentration $c^{*}$ on the blue region $B_{b}$ in Figure 3, and $c_{*}$ on the red region $B_{r}$. We will now see that choosing $c_{*}, c^{*}$ suitably, then the speed $|\mathbf{v}(t)|$ decreases, at least for small times.

Suppose that the ant is aligned with the direction $\mathbf{x}_{2}$, which in Figure 3 is the horizontal direction. Then, we have from 2.3 and the symmetry of the pheromone concentration that $F=\left(0, F_{2}\right)$. We wish to prove that $F_{2}<0$ at $t=0$, since, in that case (and since $\mathbf{v}_{1}=0$ ), the equations of motion give

$$
\dot{\mathbf{v}}_{2}(t)=-\mathbf{v}_{2}(t)+F_{2}(\mathbf{x}(t), \mathbf{v}(t))<0 \quad \text { at } t=0,
$$

as announced in the statement of the proposition. We have

$$
\begin{aligned}
F_{2} & =\int_{B_{b} \cup B_{r}} \mathbf{y}_{2} \frac{\mathcal{P}(t, \mathbf{x}+\mathbf{y})}{\int_{B_{b} \cup B_{r}} \mathcal{P}(t, \mathbf{x}+\mathbf{y}) d \mathbf{y}} d \mathbf{y} \\
& \leq \frac{1}{c_{*}\left|B_{b} \cup B_{r}\right|} \int_{B_{b} \cup B_{r}} \mathbf{y}_{2} \mathcal{P}(t, \mathbf{x}+\mathbf{y}) d \mathbf{y} \\
& =\frac{1}{c_{*}\left|B_{b} \cup B_{r}\right|} \int_{D} \mathbf{y}_{2}\left(c_{*}-c^{*}\right) d \mathbf{y},
\end{aligned}
$$

where (cf. 2.2.)

$$
D=B\left(\mathbf{v}, \ell, \beta=\frac{\pi}{2}-\theta\right)
$$


is the circular sector with half-angle $\pi / 2-\theta$. Using 2.14 and 2.15) we find

$$
F_{2}=\frac{2}{3} \ell \frac{c_{*}-c^{*}}{c_{*}} \cdot \frac{\cos \theta}{\frac{\pi}{2}+\theta} .
$$

Therefore, to ensure that $F_{2}<0$, it is sufficient to have

$$
\frac{2}{3} \ell \frac{c_{*}-c^{*}}{c_{*}} \cdot \frac{\cos \theta}{\frac{\pi}{2}+\theta}<0 \Longleftrightarrow \frac{c_{*}}{c^{*}}<1-\cos \theta .
$$

This completes the proof of Proposition 3.2

Let us now prove that under a suitable symmetry assumption on the sensing measure $\mu$, the bounds of Proposition 3.1 still hold for the model with a general, symmetric, sensing measure 2.9. Note that this result holds, in particular, for the two-point sensing model (2.13).

Proposition 3.3. Suppose that the sensing half-angle $\beta$ satisfies $0<\beta<\pi / 2$. Let $0<c_{*}<c^{*}$ be the pheromone detection thresholds in $(2.8)$, and let $\mathcal{P}_{e}$ be any effective pheromone concentration - cf. 2.7). Suppose that the sensing measure $\mu \in \mathcal{M}(B)$ is symmetric with respect to the vector $\mathbf{v}$. Then, there exist constants $C_{1}, C_{2}>0$, depending only on $\ell, c_{*}, c^{*}, \beta, \mu$ and the initial data, such that for all $t \geq 0$

$$
\min \left(|\mathbf{v}(0)|, C_{1}\right) \leq|\mathbf{v}(t)| \leq \max \left(|\mathbf{v}(0)|, C_{2}\right)
$$

where $\mathbf{v}(t)$ is a solution to 2.1, 2.9.

Proof. First, note that the bound on $\left|F_{\mu}\right|,(3.2)$, remains valid for any $\mu$. Therefore, the first part of the proof of Proposition 3.1 still holds, proving the upper bound in 3.6.

The lower bound is proved in a similar way to Proposition 3.1. Recall from its proof that we only need to have the estimate $(3.4)$, or

$$
F_{\mu} \cdot \mathbf{v} \geq C|\mathbf{v}|
$$

for some $C>0$. Consider the numerator in 2.9. We have just as in Proposition 3.1

$$
\lambda \mathbf{v} \cdot \int_{B} \mathbf{y} \mathcal{P}_{e}(t, \mathbf{x}+\mathbf{y}) d \mu(\mathbf{y}) \geq \lambda c_{*} \mathbf{v} \cdot \int_{B} \mathbf{y} d \mu(\mathbf{y}),
$$

since the functions $\mathcal{P}_{e}(t, \mathbf{x}+\mathbf{y})-c_{*}$ and $\mathbf{v} \cdot \mathbf{y} \geq 0$ are nonnegative (because $\beta<\pi / 2$ ). From the symmetry of $\mu$ with respect to $\mathbf{v}$, and, again from $\beta<\pi / 2$, we see that the vector $\int_{B} \mathbf{y} d \mu(\mathbf{y})$ is a positive multiple of $\mathbf{v}$, and so for some $C>0$

$$
\lambda \mathbf{v} \cdot \int_{B} \mathbf{y} \mathcal{P}_{e}(t, \mathbf{x}+\mathbf{y}) d \mu(\mathbf{y}) \geq C|\mathbf{v}| .
$$

For the denominator in 2.9 , we have simply

$$
\int_{B} \mathcal{P}_{e}(t, \mathbf{x}+\mathbf{y}) d \mu(\mathbf{y}) \leq \mu(B) c^{*} .
$$


With the two previous estimates, we see that (3.7) holds, which concludes the proof of 3.6 .

Just as in the case of Proposition 3.2 , one can prove that when $\beta>\pi / 2$, then the speed can decrease. We omit the proof since it is similar to the proof of Proposition 3.2

Proposition 3.4. Suppose that the sensing half-angle satisfies $\beta>\pi / 2$, and that the measure $\mu$ has mass on the region behind the ant (see Figure 3). Then, for any initial data, there exist pheromone detection thresholds $0<c_{*}<c^{*}$ and effective pheromone concentrations such that $\frac{d}{d t}|\mathbf{v}(t)|_{\left.\right|_{t=0}}<0$.

The previous a priori estimates allow us to prove a well-posedness result using the classical theorems for ordinary differential equations. We omit the proof for the sake of brevity.

Lemma 3.5. Let the sensing area $B$ be defined in $(2.2)$, with sensing half-angle $\beta$ in $(0, \pi / 2)$. Consider an effective pheromone $\mathcal{P}_{e}$ in $(2.6)$, and let the measure $\mu$ have the properties stated in Section 2.3. Then, the function $F_{\mu}: \mathbb{R}^{4} \rightarrow \mathbb{R}^{2}$,

$$
(\mathbf{x}, \mathbf{v}) \mapsto F_{\mu}(\mathbf{x}, \mathbf{v})=\lambda \int_{B(\mathbf{v})} \mathbf{y} \frac{\mathcal{P}_{e}(t, \mathbf{x}+\mathbf{y})}{\int_{B(\mathbf{v})} \mathcal{P}_{e}(t, \mathbf{x}+\mathbf{y}) d \mu(\mathbf{y})} d \mu(\mathbf{y}),
$$

is locally Lipschitz continuous and uniformly bounded over $\mathbb{R}^{4}$. Therefore, the solutions of 2.1],2.9 exist, are unique, and globally defined in time.

\subsection{Stability of trail following for a homogeneous trail}

In this section we will give a trail-like pheromone distribution depending on one spatial direction only, and consider the solution of the system (2.1),2.7) which corresponds to following along the middle of the trail. We will check that, under a symmetry assumption on the trail, this solution is linearly stable, for both models 2.7 and 2.13. Different conditions on the profile of the pheromone trail are needed in each case. Furthermore, we find a Lyapunov function for the flow, which gives the asymptotic stability of the trail-following solution.

Definition 3.6. Let $c_{*}, c^{*}$ be the pheromone detection thresholds introduced in Section 2.2. We say that $\phi: \mathbb{R} \rightarrow\left[c_{*}, c^{*}\right]$ is a trail profile if it satisfies the following properties:

1. $\phi$ is a function of the first spatial variable $\mathbf{x}_{1}$ only;

2. $\phi$ is even and smooth except possibly at $x=0$.

Given a trail profile $\phi$, we trivially extend it to $\mathbb{R}^{2}$ to obtain a trail $\phi\left(\mathbf{x}_{1}, \mathbf{x}_{2}\right)$, which we still denote by the same letter. A trail $\phi$ can be seen as a straight, symmetrical pheromone trail running along the $\mathbf{x}_{2}$ direction. A typical example is to take a (physical) pheromone distribution such as $\phi\left(\mathbf{x}_{1}\right)=a e^{-b\left|\mathbf{x}_{1}\right|}$ (for some 
constants $a, b>0)^{2}$ and, according to 2.6 and $(2.8)$, truncate this function by $c_{*}$ and $c^{*}$ in order to obtain an effective pheromone distribution. More generally, we already assume that the pheromone distribution takes values between $c_{*}$ and $c^{*}$ only.

With such a pheromone trail, the desired velocity (2.7) is independent of $\mathbf{x}_{2}$ :

$$
F=F\left(\mathbf{x}_{1}, \mathbf{v}_{1}, \mathbf{v}_{2}\right)=\lambda \int_{B} \mathbf{y} \frac{\phi\left(\mathbf{x}_{1}+\mathbf{y}_{1}\right) d \mathbf{y}}{\int_{B} \phi\left(\mathbf{x}_{1}+\mathbf{y}_{1}\right) d \mathbf{y}},
$$

with $\mathbf{y}=\left(\mathbf{y}_{1}, \mathbf{y}_{2}\right)$, and $B=B(\mathbf{v})$ defined in 2.2$)$. From now on, when we write the desired velocity $F$ depending on 3 variables only, we mean $\left(\mathbf{x}_{1}, \mathbf{v}_{1}, \mathbf{v}_{2}\right)$.

\subsubsection{Stability for the model (2.7)}

We consider first the model with desired velocity 2.7). We recall here the system 2.1,

$$
\left\{\begin{array}{l}
\dot{\mathbf{x}}_{1}=\mathbf{v}_{1} \\
\dot{\mathbf{x}}_{2}=\mathbf{v}_{2} \\
\dot{\mathbf{v}}_{1}=-\frac{1}{\tau}\left(\mathbf{v}_{1}-F_{1}\right) \\
\dot{\mathbf{v}}_{2}=-\frac{1}{\tau}\left(\mathbf{v}_{2}-F_{2}\right)
\end{array}\right.
$$

We will prove the linear stability of the solution

$$
\left(\mathbf{x}_{1}, \mathbf{x}_{2}, \mathbf{v}_{1}, \mathbf{v}_{2}\right)=\left(0, \mathbf{x}_{2}(0)+\mathbf{v}_{2}^{*} t, 0, \mathbf{v}_{2}^{*}\right)
$$

of 3.9 , where the asymptotic speed $\mathbf{v}_{2}^{*}$ is defined by

$$
F\left(0,0, \mathbf{v}_{2}^{*}\right)=\left(0, \mathbf{v}_{2}^{*}\right) \text {. }
$$

This solution corresponds to an ant following exactly along the crest of the pheromone trail with velocity $\mathbf{v}_{2}$. First, to see that indeed $(3.10)$ is a solution to 3.9 , the only nontrivial aspect is to note that for any $\alpha>0$, the vector $F(0,0, \alpha)=C \iint_{B(0, \alpha)} \mathbf{y} \phi\left(\mathbf{y}_{1}\right) d \mathbf{y}$ is parallel to $(0, \alpha)$ by the symmetry of $\phi$, and so $F_{1}(0,0, \alpha)=0$ for all time. The explicit expression for $\mathbf{v}_{2}^{*}>0$ can be computed easily:

$$
\mathbf{v}_{2}^{*}=\lambda \int_{B(0,1)} \mathbf{y}_{2} \frac{\phi\left(\mathbf{y}_{1}\right) d \mathbf{y}}{\int_{B(0,1)} \phi\left(\mathbf{y}_{1}\right) d \mathbf{y}} .
$$

We establish the following stability results:

Theorem 3.7 (Linear stability for a fixed, homogenoeous pheromone trail solution). Suppose that the sensing half-angle $\beta$ is in $(0, \pi / 2)$. Consider a pheromone trail given by Definition 3.6 and suppose that for all $0<x \leq \ell$, we have

$$
\phi(x)<\frac{1}{x} \int_{0}^{x} \phi(y) d y
$$

\footnotetext{
${ }^{2}$ Note that $\phi\left(\mathbf{x}_{1}, \mathbf{x}_{2}\right)$ is a steady-state for the diffusion equation with evaporation having as source a Dirac delta concentrated at the line $\mathbf{x}_{1}=0$.
} 
(in particular, (3.12 will hold if $\phi$ is strictly decreasing for $0<x \leq \ell$ ). Then, the solution (3.10) of 2.1, 2.7) is linearly stable.

Theorem 3.8 (Lyapunov function). Let $\mathbf{x}_{2}(0), \mathbf{v}_{2}(0)>0$. Let $\mathcal{F}: \mathbb{R} \rightarrow \mathbb{R}_{0}^{+}$be defined by

$$
\mathcal{F}(z)=-\int_{0}^{z} F_{1}(s, 0,1) d s
$$

(see (3.8)). Suppose that for some time $t_{0}$ we have $\left(\mathbf{x}\left(t_{0}\right), \mathbf{v}\left(t_{0}\right)\right)$ sufficiently close to the solution 3.10. Then, in the same conditions as Theorem 3.7, the function

$$
0 \leq L\left(\mathbf{x}_{1}, \mathbf{v}_{1}\right):=\mathbf{v}_{1}^{2}+2 \mathcal{F}\left(\mathbf{x}_{1}\right)
$$

decreases along trajectories $\mathbf{x}(t), \mathbf{v}(t)$ of 3.9 . Moreover,

$$
\lim _{t \rightarrow \infty}\|(\mathbf{x}(t), \mathbf{v}(t))-3.10\|=0 .
$$

Proof of Theorem 3.7. During this proof we omit $\lambda$ and $\tau$ since it does not change the result. Throughout the proof, we use the notation $B\left(\mathbf{v}_{1}, \mathbf{v}_{2}\right)$ to mean that the sensing area is centered on the vector $\mathbf{v}=\left(\mathbf{v}_{1}, \mathbf{v}_{2}\right)$. Recall that, since $B$ only depends on the direction of the velocity vector, then $B\left(0, \mathbf{v}_{2}\right)=B(0,1)$ for all $\mathbf{v}_{2}>0$.

Considering any solution of $2.1,(2.7)$, then from uniqueness, we can deduce that $\mathbf{v}_{2}(t)$ stays strictly positive for all $t>0$, if $\mathbf{v}_{2}(0)>0$. This is because $\left(\mathbf{x}_{1}, \mathbf{x}_{2}, \mathbf{v}_{1}, \mathbf{v}_{2}\right)=\left(\mathbf{x}_{1}(0) \pm t \overline{\mathbf{v}}_{1}, \mathbf{x}_{2}(0), \pm \overline{\mathbf{v}}_{1}, 0\right)$ are solutions for an appropriate constant $\overline{\mathbf{v}}_{1}$. Note that this is only possible due to the evenness of $\phi$, and that these solutions are orthogonal to the trail. Therefore, $t \mapsto \mathbf{x}_{2}(t)$ is strictly monotone and $\mathbf{x}_{2}(t)$ can be used as a new time variable. Thus, in the new variable, the system 3.9 becomes

$$
\left\{\begin{array}{l}
\dot{\mathbf{x}}_{1}=\frac{\mathbf{v}_{1}}{\mathbf{v}_{2}} \\
\dot{\mathbf{v}}_{1}=-\frac{\mathbf{v}_{1}}{\mathbf{v}_{2}}+\frac{F_{1}}{\mathbf{v}_{2}} \\
\dot{\mathbf{v}}_{2}=-1+\frac{F_{2}}{\mathbf{v}_{2}}
\end{array}\right.
$$

with $F=\left(F_{1}, F_{2}\right)$ given by $(3.8)$. The equilibrium is now $\left(\mathbf{x}_{1}, \mathbf{v}_{1}, \mathbf{v}_{2}\right)=\left(0,0, \mathbf{v}_{2}^{*}\right)$ with $\mathbf{v}_{2}^{*}$ as in (3.11), and we will linearize (3.14) around it.

We have to compute the partial derivatives of $F\left(\mathbf{x}_{1}, \mathbf{v}_{1}, \mathbf{v}_{2}\right)$ at the point $\left(0,0, \mathbf{v}_{2}^{*}\right)$. We will prove the following:

$$
\begin{aligned}
& \partial_{\mathbf{x}_{1}} F_{2}=\partial_{\mathbf{v}_{2}} F_{1}=\partial_{\mathbf{v}_{2}} F_{2}=0, \\
& \partial_{\mathbf{x}_{1}} F_{1}=-\alpha_{1}<0, \\
& \partial_{\mathbf{v}_{1}} F_{1}=\alpha_{2} \in[0,1),
\end{aligned}
$$

for some $\alpha_{1}>0, \alpha_{2} \geq 0$, where all functions are evaluated at $\left(0,0, \mathbf{v}_{2}^{*}\right)$. 
Let us start with (3.15). To prove that $\partial_{\mathbf{v}_{2}} F_{1}=\partial_{\mathbf{v}_{2}} F_{2}=0$, observe that $F$ only depends on $\mathbf{v}$ through the vector $\mathbf{v} /|\mathbf{v}|$. Therefore, when $\mathbf{x}_{1}=\mathbf{v}_{1}=0$, changing $\mathbf{v}_{2}$ does not change $\mathbf{v} /|\mathbf{v}|$ and so $F$ is also not changed.

Next, still in (3.15), we prove that $\partial_{\mathbf{x}_{1}} F_{2}=0$. We have

$$
F_{2}\left(\mathbf{x}_{1}, 0, \mathbf{v}_{2}^{*}\right)=\int_{B} \mathbf{y}_{2} \frac{\phi\left(\mathbf{x}_{1}+\mathbf{y}_{1}\right)}{\int_{B} \phi\left(\mathbf{x}_{1}+\mathbf{y}_{1}\right) d \mathbf{y}} d \mathbf{y},
$$

and so differentiating with respect to $\mathbf{x}_{1}$ and taking $\mathbf{x}_{1}=0$,

$$
\begin{aligned}
& \partial_{\mathbf{x}_{1}} F_{2}\left(0,0, \mathbf{v}_{2}^{*}\right)=\frac{1}{\left(\int_{B} \phi\left(\mathbf{y}_{1}\right) d \mathbf{y}\right)^{2}} \times \\
& \quad \times\left\{\int_{B} \mathbf{y}_{2} \phi^{\prime}\left(\mathbf{y}_{1}\right) d \mathbf{y} \int_{B} \phi\left(\mathbf{y}_{1}\right) d \mathbf{y}-\int_{B} \mathbf{y}_{2} \phi\left(\mathbf{y}_{1}\right) d \mathbf{y} \int_{B} \phi^{\prime}\left(\mathbf{y}_{1}\right) d \mathbf{y}\right\} .
\end{aligned}
$$

But $\int_{B} \phi^{\prime}\left(\mathbf{y}_{1}\right) d \mathbf{y}=0$, since $B=B(0,1)$ is symmetric in $\mathbf{y}_{1}$ and $\phi^{\prime}$ is an odd function. Therefore, $\partial_{\mathbf{x}_{1}} F_{2}=0$.

Now we prove (3.16). From

$$
F_{1}\left(\mathbf{x}_{1}, 0, \mathbf{v}_{2}^{*}\right)=\int_{B} \mathbf{y}_{1} \frac{\phi\left(\mathbf{x}_{1}+\mathbf{y}_{1}\right)}{\int_{B} \phi\left(\mathbf{x}_{1}+\mathbf{y}_{1}\right) d \mathbf{y}} d \mathbf{y}
$$

we get

$$
\begin{aligned}
& \partial_{\mathbf{x}_{1}} F_{1}\left(0,0, \mathbf{v}_{2}^{*}\right)=\frac{1}{\left(\int_{B} \phi\left(\mathbf{y}_{1}\right) d \mathbf{y}\right)^{2}} \times \\
& \quad \times\left\{\int_{B} \mathbf{y}_{1} \phi^{\prime}\left(\mathbf{y}_{1}\right) d \mathbf{y} \int_{B} \phi\left(\mathbf{y}_{1}\right) d \mathbf{y}-\int_{B} \mathbf{y}_{1} \phi\left(\mathbf{y}_{1}\right) d \mathbf{y} \int_{B} \phi^{\prime}\left(\mathbf{y}_{1}\right) d \mathbf{y}\right\} .
\end{aligned}
$$

Now note that the third and fourth integrals in the curly brackets vanish as before, while the second one is positive. For the first integral, we find

$$
\int_{B} \mathbf{y}_{1} \phi^{\prime}\left(\mathbf{y}_{1}\right) d \mathbf{y}=\int_{0}^{\ell} \int_{B_{\mathbf{y}_{2}}} \mathbf{y}_{1} \phi^{\prime}\left(\mathbf{y}_{1}\right) d \mathbf{y}_{1} d \mathbf{y}_{2} .
$$

For each $\mathbf{y}_{2} \in(0, \ell)$, integrating by parts we find

$$
\begin{aligned}
\int_{B_{\mathbf{y}_{2}}} \mathbf{y}_{1} \phi^{\prime}\left(\mathbf{y}_{1}\right) d \mathbf{y}_{1} & =\int_{-\mathbf{y}_{2} \sin \beta}^{\mathbf{y}_{2} \sin \beta} \mathbf{y}_{1} \phi^{\prime}\left(\mathbf{y}_{1}\right) d \mathbf{y}_{1}=2 \int_{0}^{\mathbf{y}_{2} \sin \beta} \mathbf{y}_{1} \phi^{\prime}\left(\mathbf{y}_{1}\right) d \mathbf{y}_{1} \\
& =-2 \int_{0}^{\mathbf{y}_{2} \sin \beta} \phi\left(\mathbf{y}_{1}\right) d \mathbf{y}_{1}+2 \mathbf{y}_{2} \sin \beta \phi\left(\mathbf{y}_{2} \sin \beta\right)<0,
\end{aligned}
$$

where the inequality comes from writing $x=\mathbf{y}_{2} \sin \beta$ in assumption (3.12). This proves (3.16). Note that we have also used the fact that $\beta<\pi / 2$ which ensures that each cross-section $B_{\mathbf{y}_{2}}$ is an interval containing zero. Still, note that if $\beta>\pi / 2$ and $\phi^{\prime}<0$, then (3.16) still follows. 
We move on to (3.17). First, we will show that $\partial_{\mathbf{v}_{1}} F_{1} \geq 0$ at $\left(0,0, \mathbf{v}_{2}^{*}\right)$. This is equivalent to showing that, for $\mathbf{v}_{1}>0$ sufficiently small, we have

$$
F_{1}\left(0,-\mathbf{v}_{1}, \mathbf{v}_{2}^{*}\right)<F_{1}\left(0,0, \mathbf{v}_{2}^{*}\right)<F_{1}\left(0, \mathbf{v}_{1}, \mathbf{v}_{2}^{*}\right) .
$$

Since $F_{1}\left(0,0, \mathbf{v}_{2}^{*}\right)=0$, we must check that

$$
F_{1}\left(0,-\mathbf{v}_{1}, \mathbf{v}_{2}^{*}\right)<0 \text { and } F_{1}\left(0, \mathbf{v}_{1}, \mathbf{v}_{2}^{*}\right)>0 .
$$

Let $\mathbf{v}_{1}$ be small enough that the angle $\theta=\arctan \left(\mathbf{v}_{1} / \mathbf{v}_{2}^{*}\right)$ satisfies $\beta+\theta<\pi / 2$ and also $\beta-\theta>0$. With this definition, we have $\mathbf{v} /|\mathbf{v}|=(\sin \theta, \cos \theta)$. Now note that the sign of

$$
F_{1}\left(0, \mathbf{v}_{1}, \mathbf{v}_{2}^{*}\right)=\int_{B\left(\mathbf{v}_{1}, \mathbf{v}_{2}^{*}\right)} \mathbf{y}_{1} \frac{\phi\left(\mathbf{y}_{1}\right)}{\int_{B\left(\mathbf{v}_{1}, \mathbf{v}_{2}^{*}\right)} \phi\left(\mathbf{y}_{1}\right) d \mathbf{y}} d \mathbf{y}
$$

is the same as the sign of $\int_{B\left(\mathbf{v}_{1}, \mathbf{v}_{2}^{*}\right)} \mathbf{y}_{1} \phi\left(\mathbf{y}_{1}\right) d \mathbf{y}$. Employing polar coordinates and (2.15), we find

$$
\int_{B\left(\mathbf{v}_{1}, \mathbf{v}_{2}^{*}\right)} \mathbf{y}_{1} \phi\left(\mathbf{y}_{1}\right) d \mathbf{y}=\int_{0}^{\ell} \int_{\theta-\beta}^{\theta+\beta} \sin \alpha \phi(r \sin \alpha) r^{2} d \alpha d r .
$$

We consider only the integral in $\alpha$. Using the symmetry of $\phi$,

$$
\begin{aligned}
\int_{\theta-\beta}^{\theta+\beta} \sin \alpha \phi(r \sin \alpha) d \alpha & =\left(\int_{\theta-\beta}^{0}+\int_{0}^{\theta+\beta}\right) \sin \alpha \phi(r \sin \alpha) d \alpha \\
& =\left(-\int_{0}^{\beta-\theta}+\int_{0}^{\theta+\beta}\right) \sin \alpha \phi(r \sin \alpha) d \alpha \\
& =\int_{\beta-\theta}^{\beta+\theta} \sin \alpha \phi(r \sin \alpha) d \alpha>0,
\end{aligned}
$$

since $[\beta-\theta, \beta+\theta] \subset(0, \pi / 2)$. Similarly, the sign of $F_{1}\left(0,-\mathbf{v}_{1}, \mathbf{v}_{2}^{*}\right)$ is given by the sign of

$$
\int_{0}^{\ell} \int_{-\theta-\beta}^{-\theta+\beta} \sin \alpha \phi(r \sin \alpha) r^{2} d \alpha d r
$$

and so by the sign of

$$
\int_{-\theta-\beta}^{-\theta+\beta} \sin \alpha \phi(r \sin \alpha) d \alpha=\int_{-\beta-\theta}^{-\beta+\theta} \sin \alpha \phi(r \sin \alpha) d \alpha<0,
$$

since $[-\beta-\theta,-\beta+\theta] \subset(-\pi / 2,0)$. This proves that $\partial_{\mathbf{v}_{1}} F_{1} \geq 0$.

To finish proving (3.17), we need to show that $\partial_{\mathbf{v}_{1}} F_{1}<1$. Define

$$
g\left(\mathbf{v}_{1}\right)=\int_{B\left(\mathbf{v}_{1}, \mathbf{v}_{2}^{*}\right)} \mathbf{y}_{1} \phi\left(\mathbf{y}_{1}\right) d \mathbf{y} .
$$


Then, as already observed, $g(0)=0$. Now differentiate 3.19 with respect to $\mathbf{v}_{1}$. We see that $\partial_{\mathbf{v}_{1}} F_{1}<1$ is equivalent to

$$
g^{\prime}(0)<\int_{B\left(0, \mathbf{v}_{2}^{*}\right)} \phi\left(\mathbf{y}_{1}\right) d \mathbf{y} .
$$

Since $g(0)=0,3.21$ will be proved if, for all sufficiently small $\mathbf{v}_{1}>0$ (the proof for $\mathbf{v}_{1}<0$ is similar) we have

$$
g\left(\mathbf{v}_{1}\right)<\mathbf{v}_{1} \int_{B\left(0, \mathbf{v}_{2}^{*}\right)} \phi\left(\mathbf{y}_{1}\right) d \mathbf{y} .
$$

Let us write $(3.22)$ in polar coordinates. Setting again $\theta>0$ as the angle of the vectors $\left(0, \mathbf{v}_{2}^{*}\right)$ and $\left(\mathbf{v}_{1}, \mathbf{v}_{2}^{*}\right)$, we can write $\mathbf{v}_{1}=\mathbf{v}_{2}^{*} \tan \theta$. Then, we want to prove

$$
\begin{aligned}
\int_{0}^{\ell} \int_{\theta-\beta}^{\theta+\beta} r^{2} \sin \alpha \phi(r \sin \alpha) d \alpha d r & <\mathbf{v}_{2}^{*} \tan \theta \int_{0}^{\ell} \int_{-\beta}^{\beta} r \phi(r \sin \alpha) d \alpha d r \\
& =\tan \theta \int_{0}^{\ell} \int_{-\beta}^{\beta} r^{2} \cos \alpha \phi(r \sin \alpha) d \alpha d r
\end{aligned}
$$

where we used the fact that $\mathbf{v}_{2}^{*}=F_{2}\left(0,0, \mathbf{v}_{2}^{*}\right)$ and $(3.18)$ in polar coordinates.

Now we consider only the integrals in $d \alpha$. Observe that from $(3.20)$ we may change the limits on the integral $\int_{\theta-\beta}^{\theta+\beta}$ to $\int_{\beta-\theta}^{\beta+\theta}$. We divide by $\theta>0$ on both sides so that the inequality we wish to prove for small $\theta$ 's recasts as

$$
\frac{1}{\theta} \int_{\beta-\theta}^{\beta+\theta} r^{2} \sin \alpha \phi(r \sin \alpha) d \alpha<\frac{\tan \theta}{\theta} \int_{-\beta}^{\beta} r^{2} \cos \alpha \phi(r \sin \alpha) d \alpha .
$$

Now, this inequality will hold for small enough $\theta>0$ if the limit as $\theta \rightarrow 0$ satisfies the same inequality, namely

$$
2 r^{2} \sin \beta \phi(r \sin \beta)<\int_{-\beta}^{\beta} r^{2} \cos \alpha \phi(r \sin \alpha) d \alpha .
$$

Canceling $2 r$ and from the parity of the integrand on the right-hand side, we get, equivalently,

$$
r \sin \beta \phi(r \sin \beta)<\int_{0}^{\beta} r \cos \alpha \phi(r \sin \alpha) d \alpha .
$$

Now make the change of variable $y=r \sin \alpha$ in the integral and set $x=r \sin \beta$. We get

$$
x \phi(x)<\int_{0}^{x} \phi(y) d y
$$

which is valid from assumption 3.12 . This shows that 3.17 holds. 
With (3.16)-3.17) in hand, we can assemble the matrix for the linearized system around the equilibrium 3.10 . Recalling that $-\alpha_{1}=\partial_{\mathbf{x}_{1}} F_{1}$ and $\alpha_{2}=$ $\partial_{\mathbf{v}_{1}} F_{1}$, we find

$$
\left(\begin{array}{ccc}
0 & 1 / \mathbf{v}_{2}^{*} & 0 \\
-\alpha_{1} / \mathbf{v}_{2}^{*} & \frac{-1+\alpha_{2}}{\mathbf{v}_{2}^{*}} & 0 \\
0 & \frac{\partial_{v_{1}} F_{2}}{\mathbf{v}_{2}^{*}} & -1 / \mathbf{v}_{2}^{*}
\end{array}\right) .
$$

The characteristic polynomial is

$$
\left(\frac{1}{\mathbf{v}_{2}^{*}}+\lambda\right)\left(-\lambda\left(\frac{1-\alpha_{2}}{\mathbf{v}_{2}^{*}}+\lambda\right)-\frac{\alpha_{1}}{\left(\mathbf{v}_{2}^{*}\right)^{2}}\right) .
$$

Linear stability will follow if all the roots have negative real part. One root is $\lambda=-\frac{1}{\mathbf{v}_{2}^{*}}<0$. The other two roots satisfy $-\lambda\left(\frac{1-\alpha_{2}}{\mathbf{v}_{2}^{*}}+\lambda\right)-\frac{\alpha_{1}}{\left(\mathbf{v}_{2}^{*}\right)^{2}}=0$ which is easily seen to have roots with negative real part, since from (3.17) we have $1-\alpha_{2}>0$. This concludes the proof of Theorem 3.7 .

Proof of Theorem 3.8. From (3.13) and (3.16) we find

$$
\mathcal{F}\left(\mathbf{x}_{1}\right)=\frac{\mathbf{x}_{1}^{2}}{2} \alpha_{1}+\mathcal{O}\left(\mathbf{x}_{1}^{3}\right)
$$

where, since $F\left(\mathbf{x}_{1}, 0, \mathbf{v}_{2}\right)$ and $\alpha_{1}$ do not depend on $\mathbf{v}_{2}$, the right-hand side is also independent of $\mathbf{v}_{2}$ (in particular, $\mathcal{F}\left(\mathbf{x}_{1}\right)$ is non-negative for small enough $\left.\left|\mathbf{x}_{1}\right|\right)$. Therefore, for small enough $\mathbf{x}_{1}$, we have, say,

$$
\frac{\alpha_{1}}{4} \mathbf{x}_{1}^{2} \leq \mathcal{F}\left(\mathbf{x}_{1}\right) \leq \alpha_{1} \mathbf{x}_{1}^{2},
$$

and we can find a constant $C>0$ such that

$$
\frac{1}{C}\left(\mathbf{v}_{1}^{2}+\mathbf{x}_{1}^{2}\right) \leq \mathbf{v}_{1}^{2}+2 \mathcal{F}\left(\mathbf{x}_{1}\right)+\leq C\left(\mathbf{v}_{1}^{2}+\mathbf{x}_{1}^{2}\right) .
$$

In other words, $\left(\mathbf{x}_{1}, \mathbf{v}_{1}\right) \mapsto \mathbf{v}_{1}^{2}+2 \mathcal{F}\left(\mathbf{x}_{1}\right)$ induces (for small enough $\left(\mathbf{x}_{1}, \mathbf{v}_{1}\right)$ ) a distance equivalent to the standard Euclidean distance.

Recall the special solution (3.10), and let

$$
\left(F_{1}^{*}\left(\mathbf{x}_{1}\right), F_{2}^{*}\left(\mathbf{x}_{1}\right)\right):=\left(F_{1}\left(\mathbf{x}_{1}, 0, \mathbf{v}_{2}\right), F_{2}\left(\mathbf{x}_{1}, 0, \mathbf{v}_{2}\right)\right) .
$$

From $(3.9), 3.13)$ we find

$$
\begin{aligned}
\frac{1}{2} \frac{d}{d t} \mathbf{v}_{1}^{2} & =-\mathbf{v}_{1}^{2}+\left(F_{1}\left(\mathbf{x}_{1}, \mathbf{v}_{1}, \mathbf{v}_{2}\right)-F_{1}^{*}\left(\mathbf{x}_{1}\right)\right) \mathbf{v}_{1}+F_{1}^{*}\left(\mathbf{x}_{1}\right) \mathbf{v}_{1} \\
& =-\mathbf{v}_{1}^{2}+\left(F_{1}\left(\mathbf{x}_{1}, \mathbf{v}_{1}, \mathbf{v}_{2}\right)-F_{1}^{*}\left(\mathbf{x}_{1}\right)\right) \mathbf{v}_{1}-\frac{d}{d t} \mathcal{F}\left(\mathbf{x}_{1}\right) .
\end{aligned}
$$

Therefore

$$
\begin{aligned}
\frac{1}{2} \frac{d}{d t} \mathbf{v}_{1}^{2}+\frac{d}{d t} \mathcal{F}\left(\mathbf{x}_{1}\right) & =-\mathbf{v}_{1}^{2}+\frac{F_{1}\left(\mathbf{x}_{1}, \mathbf{v}_{1}, \mathbf{v}_{2}\right)-F_{1}^{*}\left(\mathbf{x}_{1}\right)}{\mathbf{v}_{1}} \mathbf{v}_{1}^{2} \\
& =\mathbf{v}_{1}^{2}\left(-1+\partial_{\mathbf{v}_{1}} F_{1}\left(\mathbf{x}_{1}, \overline{\mathbf{v}}_{1}, \mathbf{v}_{2}\right)\right)
\end{aligned}
$$


for some $\overline{\mathbf{v}}_{1}$ between $\mathbf{v}_{1}$ and 0 . Now, by estimate 3.17 we have

$$
\partial_{\mathbf{v}_{1}} F_{1}\left(0,0, \mathbf{v}_{2}^{*}\right)<1 .
$$

For $\varepsilon_{0}, C>0$, define the rectangle

$$
S_{C, \varepsilon_{0}}=\left\{(a, b) \in \mathbb{R}^{2}: \max (a, b / 2 C) \leq \varepsilon_{0}\right\} .
$$

Fix $C$ to be chosen later. Then, by continuity, we conclude that for small enough $\varepsilon_{0}$, we have

$$
\sup _{\left(\mathbf{v}_{1}^{2}+2 \mathcal{F}\left(\mathbf{x}_{1}\right),\left(\mathbf{v}_{2}-\mathbf{v}_{2}^{*}\right)^{2}\right) \in S_{C, \varepsilon_{0}}} \partial_{\mathbf{v}_{1}} F_{1}\left(\mathbf{x}_{1}, \overline{\mathbf{v}}_{1}, \mathbf{v}_{2}\right)<1 .
$$

That is, $\partial_{\mathbf{v}_{1}} F_{1}$ is less than 1 if its argument is in a small enough neighborhood of the point $\left(0,0, \mathbf{v}_{2}^{*}\right)$, and this neighborhood can be taken as the set of points $\left(\mathbf{x}_{1}, \mathbf{v}_{1}, \mathbf{v}_{2}\right)$ for which $\left(\mathbf{v}_{1}^{2}+2 \mathcal{F}\left(\mathbf{x}_{1}\right),\left(\mathbf{v}_{2}-\mathbf{v}_{2}^{*}\right)^{2}\right) \in S_{C, \varepsilon_{0}}$.

Now suppose that

$$
\left(\mathbf{v}_{1}^{2}(0)+2 \mathcal{F}\left(\mathbf{x}_{1}(0)\right),\left(\mathbf{v}_{2}(0)-\mathbf{v}_{2}^{*}\right)^{2}\right) \in S_{C, \varepsilon_{0}} .
$$

We will prove that choosing $C$ conveniently, then at least for some time $t_{1}>0$ we will still have

$$
\left(\mathbf{v}_{1}^{2}\left(t_{1}\right)+2 \mathcal{F}\left(\mathbf{x}_{1}\left(t_{1}\right)\right),\left(\mathbf{v}_{2}\left(t_{1}\right)-\mathbf{v}_{2}^{*}\right)^{2}\right) \in S_{C, \varepsilon_{0}} .
$$

In that case, we have found a bounded invariant region for the flow and stability follows. Let us prove (3.28).

From (3.26), at least until some time $t_{1}>0$ we have

$$
\mathbf{v}_{1}^{2}\left(t_{1}\right)+2 \mathcal{F}\left(\mathbf{x}_{1}\left(t_{1}\right)\right)<\mathbf{v}_{1}^{2}(0)+2 \mathcal{F}\left(\mathbf{x}_{1}(0)\right) .
$$

Next, we observe that from $(3.9)$, and using $F_{2}\left(0,0, \mathbf{v}_{2}\right)=\mathbf{v}_{2}^{*}$, we obtain

$$
\begin{aligned}
\frac{1}{2} \frac{d}{d t}\left(\mathbf{v}_{2}-\mathbf{v}_{2}^{*}\right)^{2} & =-\left(\mathbf{v}_{2}-\mathbf{v}_{2}^{*}\right)^{2}+\left(F_{2}\left(\mathbf{x}_{1}, \mathbf{v}_{1}, \mathbf{v}_{2}\right)-F_{2}\left(0,0, \mathbf{v}_{2}\right)\right)\left(\mathbf{v}_{2}-\mathbf{v}_{2}^{*}\right) \\
& \leq-\frac{1}{2}\left(\mathbf{v}_{2}-\mathbf{v}_{2}^{*}\right)^{2}+\frac{1}{2}\left|F_{2}\left(\mathbf{x}_{1}, \mathbf{v}_{1}, \mathbf{v}_{2}\right)-F_{2}\left(0,0, \mathbf{v}_{2}\right)\right|^{2} \\
& \leq-\frac{1}{2}\left(\mathbf{v}_{2}-\mathbf{v}_{2}^{*}\right)^{2}+\frac{1}{2} \sup |\nabla F|^{2}\left(\left|\mathbf{v}_{1}\right|+\left|\mathbf{x}_{1}\right|\right)^{2},
\end{aligned}
$$

where we have used that $F$ is a globally Lipschitz function. So, for some appropriate constant $C_{1}>0$ depending only on the data of the problem (see $(3.25)$,

$$
\frac{d}{d t}\left(\mathbf{v}_{2}-\mathbf{v}_{2}^{*}\right)^{2} \leq-\left(\mathbf{v}_{2}-\mathbf{v}_{2}^{*}\right)^{2}+C_{1}\left(\mathbf{v}_{1}^{2}+2 \mathcal{F}\left(\mathbf{x}_{1}\right)\right) .
$$

Therefore

$$
\begin{aligned}
\left(\mathbf{v}_{2}\left(t_{1}\right)-\mathbf{v}_{2}^{*}\right)^{2} & \leq\left(\mathbf{v}_{2}(0)-\mathbf{v}_{2}^{*}\right)^{2} e^{-t_{1}}+C_{1} \int_{0}^{t_{1}}\left(\mathbf{v}_{1}^{2}(s)+2 \mathcal{F}\left(\mathbf{x}_{1}(s)\right)\right) e^{s-t_{1}} d s \\
& \leq\left(\mathbf{v}_{2}(0)-\mathbf{v}_{2}^{*}\right)^{2} e^{-t_{1}}+C_{1}\left(\mathbf{v}_{1}^{2}(0)+2 \mathcal{F}\left(\mathbf{x}_{1}(0)\right)\right)\left(1-e^{-t_{1}}\right) .
\end{aligned}
$$


We take $C=C_{1}$ in (3.27), (3.28). For the sake of readability, we now set

$$
a(t)=\mathbf{v}_{1}^{2}(t)+2 \mathcal{F}\left(\mathbf{x}_{1}(t)\right), \quad b(t)=\left(\mathbf{v}_{2}(t)-\mathbf{v}_{2}^{*}\right)^{2} .
$$

Now let us distinguish two cases. First, suppose that

$$
\max \left(a(0), b(0) / 2 C_{1}\right)=a(0) .
$$

Then, we obtain

$$
\begin{aligned}
b\left(t_{1}\right) & \leq b(0) e^{-t_{1}}+C_{1} a(0)\left(1-e^{-t_{1}}\right) \\
& \leq 2 C_{1} a(0) e^{-t_{1}}+C_{1} a(0)\left(1-e^{-t_{1}}\right)<2 C_{1} a(0) .
\end{aligned}
$$

Thus, $b\left(t_{1}\right) / 2 C_{1}<a(0)$. Also, from 3.29 we get $a\left(t_{1}\right)<a(0)$, and so

$$
\max \left(a\left(t_{1}\right), b\left(t_{1}\right) / 2 C_{1}\right)<a(0)=\max \left(a(0), b(0) / 2 C_{1}\right) .
$$

Second, suppose that

$$
\max \left(a(0), b(0) / 2 C_{1}\right)=b(0) / 2 C_{1} .
$$

Then, 3.29 gives $a\left(t_{1}\right)<a(0)$ just as before. Also, $C_{1} a(0)<2 C_{1} a(0) \leq b(0)$ and so

$$
\begin{aligned}
b\left(t_{1}\right) & <b(0) e^{-t_{1}}+b(0)\left(1-e^{-t_{1}}\right)=b(0) \\
& \Rightarrow b\left(t_{1}\right) / 2 C_{1}<b(0) / 2 C_{1} .
\end{aligned}
$$

This gives

$$
\max \left(a\left(t_{1}\right), b\left(t_{1}\right) / 2 C_{1}\right)<\max \left(a(0), b(0) / 2 C_{1}\right) .
$$

In both cases (3.30) and (3.31), we find that the function

$$
\max \left(\mathbf{v}_{1}^{2}(t)+2 \mathcal{F}\left(\mathbf{x}_{1}(t)\right),\left(\mathbf{v}_{2}(t)-\mathbf{v}_{2}^{*}(t)\right)^{2} / 2 C_{1}\right)
$$

decreases along the trajectories of system (3.9), at least for small times. Since the choice of $t_{1}$ only depends on universal constants, the argument can be repeated and we conclude asymptotic stability for initial data close enough to $\left(0,0, \mathbf{v}_{2}^{*}\right)$. The statement of Theorem 3.8 follows.

\subsubsection{Stability for the two-point model 2.13}

We now consider the two-point sensing measure $2.11,(2.12)$ with desired velocity 2.13). We will take a pheromone trail in the sense of Definition 3.6. However, relative to the statement of Theorem 3.7. the condition on the pheromone trail profile $\phi$ must be strengthened. Namely, we will assume that the pheromone profile $\phi(x)$ satisfies, for $0<x \leq \ell$,

$$
x \mapsto \phi(x) \text { is decreasing, and } x \mapsto x^{2} \phi(x) \text { is increasing. }
$$

The second condition in 3.32 states that, although (for positive $x$ ) $\phi$ is decreasing, it cannot decrease too rapidly near the origin. To see this, consider the particular example of $\phi(x)=e^{-b|x|}+c_{*}$, with $b>0$. Then, an elementary calculation shows that the condition $(3.32)$ is satisfied when $b \leq 2 / \ell$. 
Theorem 3.9 (Linear stability for a fixed pheromone trail solution with twopoint sensing measure). Suppose that the sensing half-angle $\beta$ is in $(0, \pi / 2)$, and that $v_{2}(0), x_{2}(0)>0$. Consider a pheromone trail $\phi$ given by Definition 3.6 and suppose that for all $0<x \leq \ell, \phi$ satisfies 3.32 . Then, the solution 3.10) of the system (3.9) with desired velocity 2.13 is linearly stable.

Proof. As in Theorem 3.7, the proof rests on the sign properties (3.15)-(3.17). We begin with (3.16). Recall the expression of $F$ in 2.13) and write $\mathbf{y}_{L}=$ $\left(\mathbf{y}_{L 1}, \mathbf{y}_{L 2}\right), \mathbf{y}_{R}=\left(\mathbf{y}_{R 1}, \mathbf{y}_{R 2}\right)$. We have

$$
F_{1}\left(\mathbf{x}_{1}, 0, \mathbf{v}_{2}^{*}\right)=\frac{\mathbf{y}_{L 1} \phi\left(\mathbf{x}_{1}+\mathbf{y}_{L 1}\right)+\mathbf{y}_{R 1} \phi\left(\mathbf{x}_{1}+\mathbf{y}_{R 1}\right)}{\phi\left(\mathbf{x}_{1}+\mathbf{y}_{L 1}\right)+\phi\left(\mathbf{x}_{1}+\mathbf{y}_{R 1}\right)} .
$$

Differentiating with respect to $\mathbf{x}_{1}$ we find that the sign of $\partial_{\mathbf{x}_{1}} F_{1}$ is given by the sign of

$$
\begin{aligned}
& \left(\mathbf{y}_{L 1} \phi^{\prime}\left(\mathbf{y}_{L 1}\right)+\mathbf{y}_{R 1} \phi^{\prime}\left(\mathbf{y}_{R 1}\right)\right)\left(\phi\left(\mathbf{y}_{L 1}\right)+\phi\left(\mathbf{y}_{R 1}\right)\right) \\
& \quad-\left(\mathbf{y}_{L 1} \phi\left(\mathbf{y}_{L 1}\right)+\mathbf{y}_{R 1} \phi\left(\mathbf{y}_{R 1}\right)\right)\left(\phi^{\prime}\left(\mathbf{y}_{L 1}\right)+\mathbf{y}_{R 1} \phi^{\prime}\left(\mathbf{y}_{R 1}\right)\right) .
\end{aligned}
$$

Using the evenness of $\phi$ and the oddness of $\phi^{\prime}$, as well as the fact that $\mathbf{y}_{L 1}=$ $-\mathbf{y}_{R 1}$, we find that the above expression simplifies to

$$
4 \mathbf{y}_{R 1} \phi^{\prime}\left(\mathbf{y}_{R 1}\right) \phi\left(\mathbf{y}_{R 1}\right)
$$

which is negative from the assumptions on $\phi$. This proves (3.16).

Next, we consider $\partial_{\mathbf{x}_{1}} F_{2}=0$. From 2.13 and 2.12 with $\mathbf{v}_{1}=0$ we have

$$
\mathbf{y}_{L}=\ell\left(\begin{array}{l}
-\sin \beta \\
\cos \beta
\end{array}\right), \quad \mathbf{y}_{R}=\ell\left(\begin{array}{c}
\sin \beta \\
\cos \beta
\end{array}\right)
$$

and

$$
F_{2}\left(\mathbf{x}_{1}, 0, \mathbf{v}_{2}^{*}\right)=\ell \frac{\cos \beta \phi\left(\mathbf{x}_{1}-\sin \beta\right)+\cos \beta \phi\left(\mathbf{x}_{1}+\sin \beta\right)}{\phi\left(\mathbf{x}_{1}-\sin \beta\right)+\phi\left(\mathbf{x}_{1}+\sin \beta\right)} \equiv \frac{N\left(\mathbf{x}_{1}\right)}{D\left(\mathbf{x}_{1}\right)} .
$$

Now note that

$$
\begin{aligned}
& N^{\prime}(0)=\ell \cos \beta\left(\phi^{\prime}(-\sin \beta)+\phi^{\prime}(\sin \beta)\right)=0, \\
& D^{\prime}(0)=\phi^{\prime}(-\sin \beta)+\phi^{\prime}(\sin \beta)=0 .
\end{aligned}
$$

This is sufficient to prove $\partial_{\mathbf{x}_{1}} F_{2}=0$. The proof of $\partial_{\mathbf{v}_{2}} F_{1}=0$ is similar to the one in Theorem 3.7

Lastly, we turn to the proof of (3.17). From $(2.13)$ we find

$$
F_{1}\left(0, \mathbf{v}_{1}, \mathbf{v}_{2}^{*}\right)=\frac{\mathbf{y}_{L 1} \phi\left(\mathbf{y}_{L 1}\right)+\mathbf{y}_{R 1} \phi\left(\mathbf{y}_{R 1}\right)}{\phi\left(\mathbf{y}_{L 1}\right)+\phi\left(\mathbf{y}_{R 1}\right)} .
$$

Let us write the expressions of $\mathbf{y}_{L}, \mathbf{y}_{R}$. Let $\theta=\arctan \left(\mathbf{v}_{1} / \mathbf{v}_{2}^{*}\right)$, so for small $\mathbf{v}_{1}$, $\theta$ is small. According to 2.12 with $\Theta=\pi / 2+\theta$,

$$
\begin{aligned}
& \mathbf{y}_{L}=\ell\left(\begin{array}{l}
\cos (\pi / 2+\theta+\beta) \\
\sin (\pi / 2+\theta+\beta)
\end{array}\right)=\ell\left(\begin{array}{c}
-\sin (\theta+\beta) \\
\cos (\theta+\beta)
\end{array}\right), \\
& \mathbf{y}_{R}=\ell\left(\begin{array}{l}
\cos (\pi / 2+\theta-\beta) \\
\sin (\pi / 2+\theta-\beta)
\end{array}\right)=\ell\left(\begin{array}{l}
-\sin (\theta-\beta) \\
\cos (\theta-\beta)
\end{array}\right) .
\end{aligned}
$$


Therefore, using the evenness of $\phi$,

$$
F_{1}\left(0, \mathbf{v}_{1}, \mathbf{v}_{2}^{*}\right)=\frac{-\ell(\sin (\theta-\beta) \phi(\ell \sin (\theta-\beta))+\sin (\theta+\beta) \phi(\ell \sin (\theta+\beta)))}{\phi(\ell \sin (\theta-\beta))+\phi(\ell \sin (\theta+\beta))} .
$$

We must compute $\left.\partial_{\mathbf{v}_{1}} F_{1}\left(0, \mathbf{v}_{1}, \mathbf{v}_{2}^{*}\right)\right|_{\mathbf{v}_{1}=0}$. Write the above expression as

$$
F_{1}\left(0, \mathbf{v}_{1}, \mathbf{v}_{2}^{*}\right)=\frac{N\left(\mathbf{v}_{1}\right)}{D\left(\mathbf{v}_{1}\right)}
$$

and note that $N(0)=0$. Therefore,

$$
\left.\partial_{\mathbf{v}_{1}}\left(\frac{N\left(\mathbf{v}_{1}\right)}{D\left(\mathbf{v}_{1}\right)}\right)\right|_{\mathbf{v}_{1}=0}<1 \Longleftrightarrow N^{\prime}(0)<D(0) .
$$

We have $\left.\theta^{\prime}\left(\mathbf{v}_{1}\right)\right|_{\mathbf{v}_{1}=0}=\left(\mathbf{v}_{2}^{*}\right)^{-1}$, and so an easy computation gives

$$
N^{\prime}(0)=-2 \frac{\ell}{\mathbf{v}_{2}^{*}} \cos \beta\left(\phi(\ell \sin \beta)+\ell \sin \beta \phi^{\prime}(\ell \sin \beta)\right) .
$$

On the other hand,

$$
D(0)=2 \phi(\ell \sin \beta)
$$

Now we compute $\mathbf{v}_{2}^{*}$ as the value for which $\left(0, \mathbf{v}_{2}^{*}\right)=F\left(0,0, \mathbf{v}_{2}^{*}\right)$, with $F$ given by (2.13). We find with the evenness of $\phi$ and $\mathbf{y}_{R 1}=-\mathbf{y}_{L 1}$ for $\mathbf{v}_{1}=0$ in (2.12),

$$
\mathbf{v}_{2}^{*}=\frac{\mathbf{y}_{L 2} \phi\left(\mathbf{y}_{L 1}\right)+\mathbf{y}_{R 2} \phi\left(\mathbf{y}_{R 1}\right)}{\phi\left(\mathbf{y}_{L 1}\right)+\phi\left(\mathbf{y}_{R 1}\right)}=\ell \cos \beta .
$$

Therefore,

$$
N^{\prime}(0)<D(0) \Longleftrightarrow-\phi(\ell \sin \beta)-\ell \sin \beta \phi^{\prime}(\ell \sin \beta)<\phi(\ell \sin \beta) .
$$

This will be fulfilled if for $x \leq \ell$,

$$
-\phi(x)-x \phi^{\prime}(x)<\phi(x),
$$

which is equivalent to the second condition of 3.32 . This finishes the proof of Theorem 3.9.

Remark 3.10. We wish to emphasize that Theorem 3.8, establishing the existence of a Lyapunov function for the flow, remains valid in the case of the two-point sensing model. Indeed, its proof relies entirely on the structure of the differential equations and on the properties $(3.15)-(3.17)$, which also hold for the two-point sensing model. 


\subsection{A stability result for an inhomogeneous trail}

The trail-like pheromone distribution which was considered in the previous section allowed us to obtain stability results (Theorems 3.7, 3.8 and 3.9. This was possible thanks to the fact that the trail pheromone only depended on one spatial direction, and so was completely described by the trail profile $\phi$ (cf. Definition 3.6 . However, in nature trails are not perfectly uniform, and so it would be interesting to consider more general pheromone distributions allowing for some variation in the direction parallel to the trail.

In this section, we provide a partial result in this direction. We consider a pheromone distribution of the form $\phi\left(\mathbf{x}_{1}\right) \psi\left(\mathbf{x}_{2}\right)$, where $\phi$ is (as in Definition 3.6) a trail profile, and $\psi\left(\mathbf{x}_{2}\right)$ represents the variation of the trail along its length. Under certain assumptions on $\phi$ and $\psi$, we will prove that there still exists a solution which corresponds to following the trail along its crest. Moreover, we will show that if the ant is traveling along the center of the trail, then a small perturbation in its direction will give rise to a desired velocity lying between the perturbation and the direction of the trail. In other words, if the ant deviates slightly from the center of the trail, it will desire to align itself with the trail again.

To model a pheromone trail running along the direction $\mathbf{x}_{2}$ but with some variation in the $\mathbf{x}_{1}$ direction, we need to assume some more restrictive properties for the trail profile $\phi$, when compared to the results in the previous section. Specifically, we consider a pheromone distribution of the form $\phi\left(\mathbf{x}_{1}\right) \psi\left(\mathbf{x}_{2}\right)$, satisfying the following properties:

1. The trail profile $\phi(y)$ is even, smooth on $[0,+\infty)$, and strictly decreasing for $0 \leq y \leq \ell$, so that

$$
\inf _{[0, \ell]}\left|\phi^{\prime}\right|>0 ;
$$

2. $\psi$ (the variation along the trail direction) is smooth, bounded, and bounded away from zero,

$$
\inf _{\mathbb{R}} \psi>0 \text { uniformly; }
$$

3. We suppose that

$$
c_{*} \leq \phi\left(\mathbf{x}_{1}\right) \psi\left(\mathbf{x}_{2}\right) \leq c^{*}, \quad \forall\left(\mathbf{x}_{1}, \mathbf{x}_{2}\right) \in \mathbb{R}^{2},
$$

where $c_{*}, c^{*}$ are the pheromone detection thresholds introduced in Section 2.2 ,

A typical trail is shown in Figure 4

The first step towards our stability result is to observe that there is a solution of the system 2.1$], 2.7)$, with effective pheromone $\mathcal{P}_{e}\left(\mathbf{x}_{1}, \mathbf{x}_{2}\right)=\phi\left(\mathbf{x}_{1}\right) \psi\left(\mathbf{x}_{2}\right)$ (with $\phi$ and $\psi$ verifying (3.33)-(3.35), which corresponds to following along the crest, or middle, of the trail, in the $\mathbf{x}_{2}$ direction. 


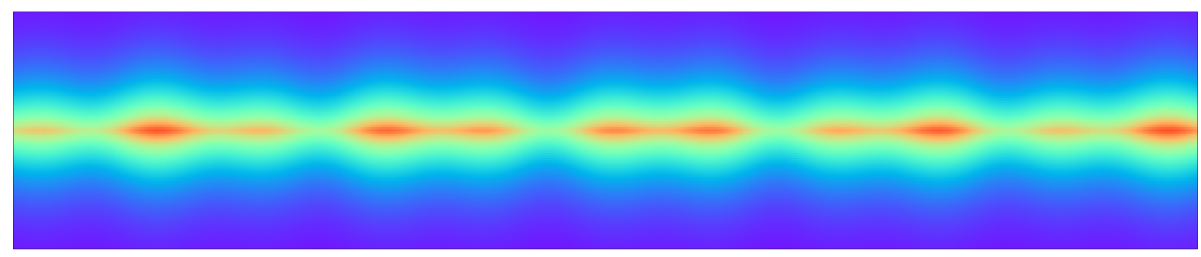

Figure 4: A typical trail of the form $\phi\left(\mathbf{x}_{1}\right) \psi\left(\mathbf{x}_{2}\right) . \mathbf{x}_{2}$ is the horizontal direction.

Lemma 3.11. Consider an initial data $\left(0, \mathbf{x}_{2}^{0}, 0, \mathbf{v}_{2}^{0}\right)$ with $\mathbf{v}_{2}^{0}>0$ and suppose that the sensing half-angle $\beta$ is in $(0, \pi / 2)$. There exists a solution $\left(0, \mathbf{x}_{2}^{*}(t), 0, \mathbf{v}_{2}^{*}(t)\right)$ of 2.1),2.7) with a pheromone trail given by 3.33)-3.35, such that

$$
\left\{\begin{array}{l}
\dot{\mathbf{x}}_{2}^{*}=\mathbf{v}_{2}^{*} \\
\dot{\mathbf{v}}_{2}^{*}=-\frac{1}{\tau}\left(\mathbf{v}_{2}^{*}-F_{2}\left(\mathbf{x}_{2}^{*}\right)\right) .
\end{array}\right.
$$

Proof. Just note that with $F$ defined in 2.7 , then $F_{2}\left(0, \mathbf{x}_{2}^{*}(t), 0, \mathbf{v}_{2}^{*}(t)\right)$ only depends on $\mathbf{x}_{2}$, since if $\mathbf{v}_{1}=0$, then $B$ does not depend on the second component $\mathbf{v}_{2}$. The solution exists if $\beta<\pi / 2$, since in that case Lemma 3.5 applies.

The stability result is as follows.

Theorem 3.12. Suppose that the sensing half-angle is in $(0, \pi / 2)$. Consider a pheromone trail $\phi\left(\mathbf{x}_{1}\right) \psi\left(\mathbf{x}_{2}\right)$ verifying the properties 3.33$)-(3.35)$. In addition, suppose that the variation along the trail, $\psi$, is sufficiently small in the sense that

$$
\frac{c^{*}\left\|\psi^{\prime}\right\|_{\infty}}{\inf _{\mathbb{R}} \psi}\left\{\frac{8}{\sin \beta}\left(\frac{c^{*}}{c_{*}}\right)^{2}+\tan \beta\right\}<\inf _{[0, \ell]}\left|\phi^{\prime}\right| .
$$

Then, we have

$$
\partial_{\mathbf{v}_{2}} F_{1}=\partial_{\mathbf{v}_{2}} F_{2}=0, \quad \partial_{\mathbf{v}_{1}} F_{2}=0, \quad \partial_{\mathbf{v}_{1}} F_{1}<1
$$

along the solution $\left(0, \mathbf{x}_{2}^{*}(t), 0, \mathbf{v}_{2}^{*}(t)\right)$ given in Lemma 3.11 .

Remark 3.13. 1. We interpret this result, in a heuristic way, as follows: From the symmetry of the trail, the desired velocity $F$ along the solution in Lemma 3.11 has zero first component. Consider a small perturbation in the value of the velocity vector of that solution, of the form

$$
\mathbf{v}_{\epsilon}=\mathbf{v}^{*}+\left(\epsilon_{1}, \pm \epsilon_{2}\right)=\left(\epsilon_{1}, \mathbf{v}_{2}^{*} \pm \epsilon_{2}\right) .
$$

If 3.38 holds, then the perturbed desired velocity $F\left(\mathbf{v}_{\epsilon}\right)$ satisfies

$$
\begin{aligned}
F_{1}\left(\mathbf{v}_{\epsilon}\right)=F_{1}\left(0, \mathbf{x}_{2}^{*}, \epsilon_{1}, \mathbf{v}_{2}^{*} \pm \epsilon_{2}\right) & =0+\epsilon_{1} \partial_{\mathbf{v}_{1}} F_{1}\left(0, \mathbf{x}_{2}^{*}, 0, \mathbf{v}_{2}^{*}\right)+\mathcal{O}\left(\epsilon_{1}^{2}+\epsilon_{2}^{2}\right) \\
& <\epsilon_{1}+\mathcal{O}\left(\epsilon_{1}^{2}+\epsilon_{2}^{2}\right),
\end{aligned}
$$




$$
F_{2}\left(\mathbf{v}_{\epsilon}\right)=F_{2}\left(0, \mathbf{x}_{2}^{*}, \epsilon_{1}, \mathbf{v}_{2}^{*} \pm \epsilon_{2}\right)=F_{2}\left(0, \mathbf{x}_{2}^{*}, 0, \mathbf{v}_{2}^{*}\right)+\mathcal{O}\left(\epsilon_{1}^{2}+\epsilon_{2}^{2}\right) .
$$

From the dynamics of the system (2.1), we expect the velocity vector $\mathbf{v}_{\epsilon}$ to relax to the desired velocity $F_{\epsilon}$. But the previous inequalities, discarding the second order terms, show that $F_{\epsilon}$ is actually between $\mathbf{v}^{*}$ and $\mathbf{v}_{\epsilon}$, for small enough $\epsilon_{i}$. Therefore, the perturbed velocity $\mathbf{v}_{\epsilon}$ will tend to relax to the trail-following direction of $\mathbf{v}^{*}$; see Figure 5

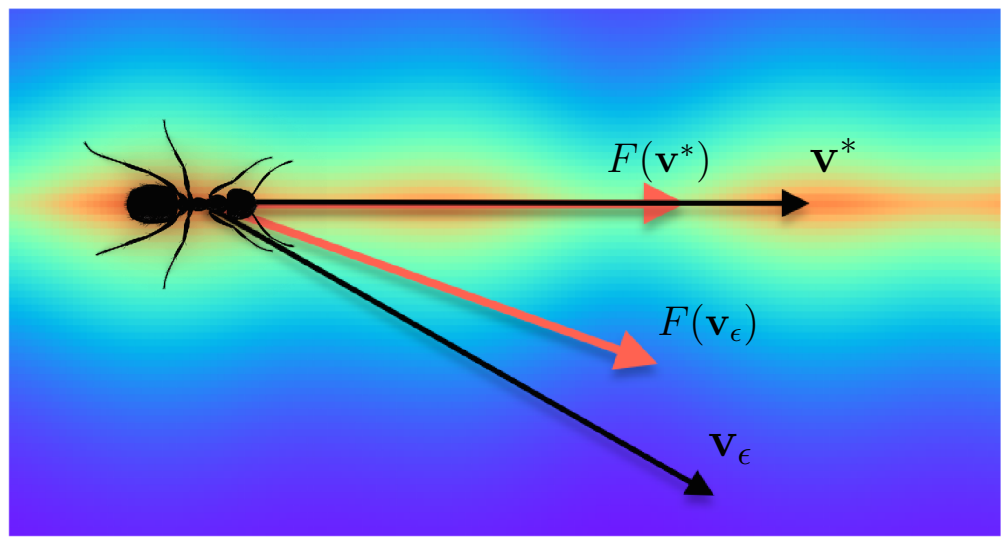

Figure 5: Illustration of the result of Theorem 3.12

2. For a fixed $\phi$, the condition (3.37) may be viewed either as saying that $\psi$ must remain sufficiently far from zero (due to the inf $\psi$ term), or that the "frequency" of $\psi$ remains small (due to the $\left\|\psi^{\prime}\right\|_{\infty}$ term). Alternatively, with $\psi$ fixed, (3.37) can be seen as a condition of sufficiently fast decrease of the trail profile $\phi$ near the center of the trail.

Proof of Theorem 3.12. Recall that for $i=1,2$,

$$
F_{i}\left(0, \mathbf{x}_{2}^{*}, \mathbf{v}_{1}, \mathbf{v}_{2}^{*}\right)=\int_{B\left(\mathbf{v}_{1}, \mathbf{v}_{2}^{*}\right)} \mathbf{y}_{i} \frac{\phi\left(\mathbf{y}_{1}\right) \psi\left(\mathbf{x}_{2}^{*}+\mathbf{y}_{2}\right)}{\int_{B\left(\mathbf{v}_{1}, \mathbf{v}_{2}^{*}\right)} \phi\left(\mathbf{y}_{1}\right) \psi\left(\mathbf{x}_{2}^{*}+\mathbf{y}_{2}\right) d \mathbf{y}} d \mathbf{y} .
$$

First, observe that the argument to prove $\partial_{\mathbf{v}_{2}} F_{1}=\partial_{\mathbf{v}_{2}} F_{2}=0$ is the same as in the proof of (3.15). The property $\partial_{\mathbf{v}_{1}} F_{2}=0$ follows from 3.39 with $i=2$ and observing that this expression is even with respect to $\mathbf{v}_{1}$ (by the symmetry of the trail). Therefore, since $\mathbf{v}_{1} \mapsto F_{2}\left(0, \mathbf{x}_{2}^{*}, \mathbf{v}_{1}, \mathbf{v}_{2}^{*}\right)$ is a smooth enough function of $\mathbf{v}_{1}$ (at least for small $\mathbf{v}_{1}$ ), we have $\partial_{\mathbf{v}_{1}} F_{2}=0$.

To prove $\partial_{\mathbf{v}_{1}} F_{1}<1$, the starting point is the same as in the proof of property (3.17) in Theorem 3.7. We define

$$
g\left(\mathbf{v}_{1}\right)=\int_{B\left(\mathbf{v}_{1}, \mathbf{v}_{2}^{*}\right)} \mathbf{y}_{1} \phi\left(\mathbf{y}_{1}\right) \psi\left(\mathbf{x}_{2}^{*}+\mathbf{y}_{2}\right) d \mathbf{y} .
$$


Then, as already observed, $g(0)=0$ due to the symmetry of $\phi$. Now differentiate 3.39 with respect to $\mathbf{v}_{1}$. We see that $\partial_{v_{1}} F_{1}<1$ is equivalent to

$$
g^{\prime}(0)<\int_{B\left(0, \mathbf{v}_{2}^{*}\right)} \phi\left(\mathbf{y}_{1}\right) \psi\left(\mathbf{x}_{2}^{*}+\mathbf{y}_{2}\right) d \mathbf{y} .
$$

Since $g(0)=0,3.40$ will be proved if, for all sufficiently small $\mathbf{v}_{1}>0$ (the proof for $\mathbf{v}_{1}<0$ is similar) we have

$$
g\left(\mathbf{v}_{1}\right)<\mathbf{v}_{1} \int_{B\left(0, \mathbf{v}_{2}^{*}\right)} \phi\left(\mathbf{y}_{1}\right) \psi\left(\mathbf{x}_{2}^{*}+\mathbf{y}_{2}\right) d \mathbf{y} .
$$

Let us write (3.41) in polar coordinates. Setting again $\theta>0$ as the angle of the vectors $\left(0, \mathbf{v}_{2}^{*}\right)$ and $\left(\mathbf{v}_{1}, \mathbf{v}_{2}^{*}\right)$, we can write $\mathbf{v}_{1}=\mathbf{v}_{2}^{*} \tan \theta$. Then, we want to prove

$$
\begin{aligned}
& \int_{0}^{\ell} \int_{\theta-\beta}^{\theta+\beta} r^{2} \sin \alpha \phi(r \sin \alpha) \psi\left(\mathbf{x}_{2}^{*}+r \cos \alpha\right) d \alpha d r \\
& \quad<\mathbf{v}_{2}^{*} \tan \theta \int_{0}^{\ell} \int_{-\beta}^{\beta} r \phi(r \sin \alpha) \psi\left(\mathbf{x}_{2}^{*}+r \cos \alpha\right) d \alpha d r
\end{aligned}
$$

Now, the proof differs from Theorem 3.7 since here we do not have $\mathbf{v}_{2}^{*}=F_{2}$. We argue as follows.

Write $\mathbf{v}_{2}^{*}=\mathbf{v}_{2}^{*} \pm F_{2}\left(\mathbf{x}_{2}^{*}\right)$. Then, the right-hand side in 3.42 becomes $I_{1}+I_{2}$, where

$$
\begin{gathered}
I_{1}=\left(\mathbf{v}_{2}^{*}-F_{2}\left(\mathbf{x}_{2}^{*}\right)\right) \tan \theta \int_{0}^{\ell} \int_{-\beta}^{\beta} r \phi(r \sin \alpha) \psi\left(\mathbf{x}_{2}^{*}+r \cos \alpha\right) d \alpha d r \\
I_{2}=F_{2}\left(\mathbf{x}_{2}^{*}\right) \tan \theta \int_{0}^{\ell} \int_{-\beta}^{\beta} r \phi(r \sin \alpha) \psi\left(\mathbf{x}_{2}^{*}+r \cos \alpha\right) d \alpha d r
\end{gathered}
$$

To deal with $I_{1}$, we wish to bound $\mathbf{v}_{2}^{*}-F_{2}\left(\mathbf{x}_{2}^{*}\right)$. For this, note that

$$
\frac{d}{d t}\left(\mathbf{v}_{2}^{*}-F_{2}\left(\mathbf{x}_{2}^{*}\right)\right)=-\left(\mathbf{v}_{2}^{*}-F_{2}\left(\mathbf{x}_{2}^{*}\right)\right)-\partial_{\mathbf{x}_{2}} F_{2}\left(\mathbf{x}_{2}^{*}\right) \mathbf{v}_{2}^{*} .
$$

Using an energy argument exactly like in Proposition 3.1, we see that setting $w(t)=\mathbf{v}_{2}^{*}-F_{2}\left(\mathbf{x}_{2}^{*}\right)$, and if $Q$ is such that $\left|\partial_{\mathbf{x}_{2}} F_{2}\left(\mathbf{x}_{2}^{*}\right) \mathbf{v}_{2}^{*}\right| \leq Q$, then we have $|w(t)| \leq \max (|w(0)|, Q)$. In light of this, we note first that from (3.35), (3.1) and 2.14 , we have

$$
\|\phi\|_{\infty} \leq \frac{c^{*}}{\inf _{\mathbb{R}} \psi}, \quad \int_{B} \phi\left(\mathbf{y}_{1}\right) \psi\left(\mathbf{x}_{2}^{*}+\mathbf{y}_{2}\right) d \mathbf{y} \geq \beta \ell^{2} c_{*}, \quad\left|\mathbf{v}_{2}^{*}\right| \leq \ell .
$$


Using these properties, 2.15), and (3.39), we get

$$
\begin{aligned}
\left|\partial_{\mathbf{x}_{2}} F_{2}\left(\mathbf{x}_{2}^{*}\right) \mathbf{v}_{2}^{*}\right| \leq & \frac{\left|\mathbf{v}_{2}^{*}\right|}{\left(\int_{B} \phi\left(\mathbf{y}_{1}\right) \psi\left(\mathbf{x}_{2}^{*}+\mathbf{y}_{2}\right) d \mathbf{y}\right)^{2}} \times \\
\times & \mid \int_{B} \mathbf{y}_{2} \phi\left(\mathbf{y}_{1}\right) \psi^{\prime}\left(\mathbf{x}_{2}^{*}+\mathbf{y}_{2}\right) \mathbf{v}_{2}^{*} d \mathbf{y} \int_{B} \phi\left(\mathbf{y}_{1}\right) \psi\left(\mathbf{x}_{2}^{*}+\mathbf{y}_{2}\right) d \mathbf{y} \\
& -\int_{B} \mathbf{y}_{2} \phi\left(\mathbf{y}_{1}\right) \psi\left(\mathbf{x}_{2}^{*}+\mathbf{y}_{2}\right) d \mathbf{y} \int_{B} \phi\left(\mathbf{y}_{1}\right) \psi^{\prime}\left(\mathbf{x}_{2}^{*}+\mathbf{y}_{2}\right) \mathbf{v}_{2}^{*} d \mathbf{y} \mid \\
\leq & 2 \frac{\left\|\psi^{\prime}\right\|_{\infty}}{\beta \inf _{\mathbb{R}} \psi}\left(\frac{c^{*}}{c_{*}}\right)^{2} \int_{B} \mathbf{y}_{2} d \mathbf{y} \\
= & 2 \frac{\left\|\psi^{\prime}\right\|_{\infty}}{\beta \inf _{\mathbb{R}} \psi}\left(\frac{c^{*}}{c_{*}}\right)^{2} \ell^{3} \frac{2}{3} \sin \beta .
\end{aligned}
$$

Therefore, bounding the integral in the definition of $I_{1}$ by $\ell^{2} 2 \beta c^{*}$, and supposing for simplicity's sake that $\max (|w(0)|, Q)=Q$,

$$
\begin{aligned}
\left|I_{1}\right| & \leq \ell^{3} \frac{4\left\|\psi^{\prime}\right\|_{\infty} \sin \beta}{3 \beta \inf _{\mathbb{R}} \psi}\left(\frac{c^{*}}{c_{*}}\right)^{2} \tan \theta \int_{0}^{\ell} \int_{-\beta}^{\beta} r \phi(r \sin \alpha) \psi\left(\mathbf{x}_{2}^{*}+r \cos \alpha\right) d \alpha d r \\
& \leq \ell^{5} \frac{8\left\|\psi^{\prime}\right\|_{\infty} \sin \beta}{3 \inf _{\mathbb{R}} \psi} \frac{\left(c^{*}\right)^{3}}{c_{*}^{2}} \tan \theta .
\end{aligned}
$$

In view of this, $I_{1}+I_{2}$ satisfies

$$
\begin{aligned}
I_{1}+I_{2} \geq & F_{2}\left(\mathbf{x}_{2}^{*}\right) \tan \theta \int_{0}^{\ell} \int_{-\beta}^{\beta} r \phi(r \sin \alpha) \psi\left(\mathbf{x}_{2}^{*}+r \cos \alpha\right) d \alpha d r \\
& -\ell^{5} \frac{8\left\|\psi^{\prime}\right\|_{\infty} \sin \beta}{3 \inf _{\mathbb{R}} \psi} \frac{\left(c^{*}\right)^{3}}{c_{*}^{2}} \tan \theta .
\end{aligned}
$$

Using 3.39 in polar coordinates, 3.42 becomes

$$
\begin{aligned}
\int_{0}^{\ell} & \int_{\theta-\beta}^{\theta+\beta} r^{2} \sin \alpha \phi(r \sin \alpha) \psi\left(\mathbf{x}_{2}^{*}+r \cos \alpha\right) d \alpha d r \\
& <\tan \theta \int_{0}^{\ell} \int_{-\beta}^{\beta} r^{2} \cos \alpha \phi(r \sin \alpha) \psi\left(\mathbf{x}_{2}^{*}+r \cos \alpha\right) d \alpha d r \\
& -\ell^{5} \frac{8\left\|\psi^{\prime}\right\|_{\infty} \sin \beta}{3 \inf _{\mathbb{R}} \psi} \frac{\left(c^{*}\right)^{3}}{c_{*}^{2}} \tan \theta .
\end{aligned}
$$

Now we consider only what is inside the integrals in $d r$, and note that $\frac{\ell^{3}}{3}=$ $\int_{0}^{\ell} r^{2} d r$. Observe that exactly as in 3.20 we may change the limits on the integral $\int_{\theta-\beta}^{\theta+\beta}$ to $\int_{\beta-\theta}^{\beta+\theta}$. We divide by $\theta>0$ on both sides to obtain the following 
formulation of the inequality we wish to establish,

$$
\begin{aligned}
& \frac{1}{\theta} \int_{\beta-\theta}^{\beta+\theta} r^{2} \sin \alpha \phi(r \sin \alpha) \psi\left(\mathbf{x}_{2}^{*}+r \cos \alpha\right) d \alpha \\
& <\frac{\tan \theta}{\theta} \int_{-\beta}^{\beta} r^{2} \cos \alpha \phi(r \sin \alpha) \psi\left(\mathbf{x}_{2}^{*}+r \cos \alpha\right) d \alpha \\
& \quad-\frac{\tan \theta}{\theta} \ell^{2} \frac{8\left\|\psi^{\prime}\right\|_{\infty} r^{2} \sin \beta}{\inf _{\mathbb{R}} \psi} \frac{\left(c^{*}\right)^{3}}{c_{*}^{2}} .
\end{aligned}
$$

Now, this inequality will hold for sufficiently small $\theta>0$ if the limit as $\theta \rightarrow 0$ satisfies the same inequality, namely

$$
\begin{aligned}
& 2 r^{2} \sin \beta \phi(r \sin \beta) \psi\left(\mathbf{x}_{2}^{*}+r \cos \beta\right) \\
& <\int_{-\beta}^{\beta} r^{2} \cos \alpha \phi(r \sin \alpha) \psi\left(\mathbf{x}_{2}^{*}+r \cos \alpha\right) d \alpha \\
& \quad-\ell^{2} \frac{8\left\|\psi^{\prime}\right\|_{\infty} r^{2} \sin \beta}{\inf _{\mathbb{R}} \psi} \frac{\left(c^{*}\right)^{3}}{c_{*}^{2}} .
\end{aligned}
$$

Canceling $2 r$ and from the parity of the integrand on the right-hand side, we get equivalently

$$
\begin{aligned}
& r \sin \beta \phi(r \sin \beta) \psi\left(\mathbf{x}_{2}^{*}+r \cos \beta\right) \\
& \quad<\int_{0}^{\beta} r \cos \alpha \phi(r \sin \alpha) \psi\left(\mathbf{x}_{2}^{*}+r \cos \alpha\right) d \alpha-\ell^{2} \frac{4\left\|\psi^{\prime}\right\|_{\infty} r \sin \beta}{\inf _{\mathbb{R}} \psi} \frac{\left(c^{*}\right)^{3}}{c_{*}^{2}} .
\end{aligned}
$$

Now make the change of variable $y=r \sin \alpha$ in the integral and set $x=r \sin \beta$. We get

$$
\begin{gathered}
x \phi(x) \psi\left(\mathbf{x}_{2}^{*}+\sqrt{r^{2}-x^{2}}\right)<\int_{0}^{x} \phi(y) \psi\left(\mathbf{x}_{2}^{*}+\sqrt{r^{2}-y^{2}}\right) d y \\
-x^{2} \frac{4\left\|\psi^{\prime}\right\|_{\infty}}{\sin \beta \inf _{\mathbb{R}} \psi} \frac{\left(c^{*}\right)^{3}}{c_{*}^{2}} .
\end{gathered}
$$

Note that for a general smooth function $\Phi$, the following holds:

$$
\int_{0}^{x} y \Phi^{\prime}(y) d y<0 \Longleftrightarrow x \Phi(x)<\int_{0}^{x} \Phi(y) d y .
$$

Using this fact, we find that (3.44) is equivalent to

$$
\begin{aligned}
\int_{0}^{x} y & \frac{d}{d y}\left(\phi(y) \psi\left(\mathbf{x}_{2}^{*}+\sqrt{r^{2}-y^{2}}\right)\right) d y+\int_{0}^{x} \phi(y) \psi\left(\mathbf{x}_{2}^{*}+\sqrt{r^{2}-y^{2}}\right) d y \\
& <-x^{2} \frac{4\left\|\psi^{\prime}\right\|_{\infty}}{\sin \beta \inf _{\mathbb{R}} \psi} \frac{\left(c^{*}\right)^{3}}{c_{*}^{2}} .
\end{aligned}
$$


But the left-hand side is bounded by (recall that $\phi^{\prime}<0$ )

$$
\begin{aligned}
\int_{0}^{x} y & \frac{d}{d y}\left(\phi(y) \psi\left(\mathbf{x}_{2}^{*}+\sqrt{r^{2}-y^{2}}\right)\right) d y+\int_{0}^{x} \phi(y) \psi\left(\mathbf{x}_{2}^{*}+\sqrt{r^{2}-y^{2}}\right) d y \\
& \leq \inf _{\mathbb{R}} \psi \int_{0}^{x} y \phi^{\prime}(y) d y+\frac{c^{*}}{\inf _{\mathbb{R}} \psi} \sup _{(0, x)} \frac{y}{\sqrt{r^{2}-y^{2}}}\left\|\psi^{\prime}\right\|_{\infty} \frac{x^{2}}{2} \\
& \leq \inf _{\mathbb{R}} \psi \inf _{[0, \ell]}\left|\phi^{\prime}\right| \frac{x^{2}}{2}+\frac{c^{*}}{\inf _{\mathbb{R}} \psi} \tan \beta\left\|\psi^{\prime}\right\|_{\infty} \frac{x^{2}}{2} .
\end{aligned}
$$

Therefore, canceling $x^{2}, 3.45$ will hold if

$$
-\inf _{\mathbb{R}} \psi \inf _{[0, \ell]}\left|\phi^{\prime}\right|+\frac{c^{*}}{\inf _{\mathbb{R}} \psi} \tan \beta\left\|\psi^{\prime}\right\|_{\infty}<-\frac{8\left\|\psi^{\prime}\right\|_{\infty}}{\sin \beta \inf _{\mathbb{R}} \psi} \frac{\left(c^{*}\right)^{3}}{c_{*}^{2}},
$$

which is equivalent to (3.37). This completes the proof of Theorem 3.12

\section{Influence of the sensing angle on trajectory shape}

One of the questions raised by the previous analysis is to know how the trajectory shape is influenced by the sensing angle $2 \beta$. We have already seen that the model's stability properties hold whenever $\beta<\pi / 2$, and that in that same regime the speed remains bounded away from zero. This leaves open the question of what are the properties of the solutions when the sensing area is larger than a half-circle, and also of what is the character of the decay to the equilibrium solution even in the stable regime.

Here, we address these questions by means of numerical experiments, using the model with two-point sensing 2.13). First, our simulations show that (in the stable regime at least) trajectories that follow a pheromone trail behave like overdamped oscillations in such a way that they decay to the pheromone trail as $e^{-\zeta(\beta) t}$ with $\zeta(\beta)$ behaving as

$$
\zeta(\beta) \propto \beta^{2}
$$

and with a wave number

$$
\omega(\beta) \propto \beta .
$$

Both approximations are valid for small $\beta$ values, and were tested for a large range of parameters. In Figure 6(a) we draw the profile of the solutions for different values of the sensing angle, exhibiting the stated behavior. The pheromone trail is located at $y=0$ and runs horizontally. In Figure 6(b),(c), we show the obtained values for the damping $\zeta$ and the wave number $\omega$.

Whenever the half-angle $\beta$ is larger than $\pi / 2$, trajectories do not seem to be unstable, but converge asymptotically to points located near or on the center of the trail. One such typical trajectory is presented in Figure 7 where, again, the pheromone trail runs horizontally along $y=0$. Further, by adding noise we find that the numerics seems to be robust and that this asymptotic point seems 
to be stable: we observe a random walk around this point. This implies that the ant gets trapped in a given position in space: i.e., the ant fails to explore the information clues provided by the pheromone trail. Thus, we are able to conclude (at least numerically) that $\beta$ controls the shape of trajectories: for small values the asymptotic convergence to the pheromone trail is low and with large wave length. But increasing $\beta$, the convergence is faster and the wave length shorter. For larger values of $\beta$ exceeding $\pi / 2$, ants are not able to follow the trail.
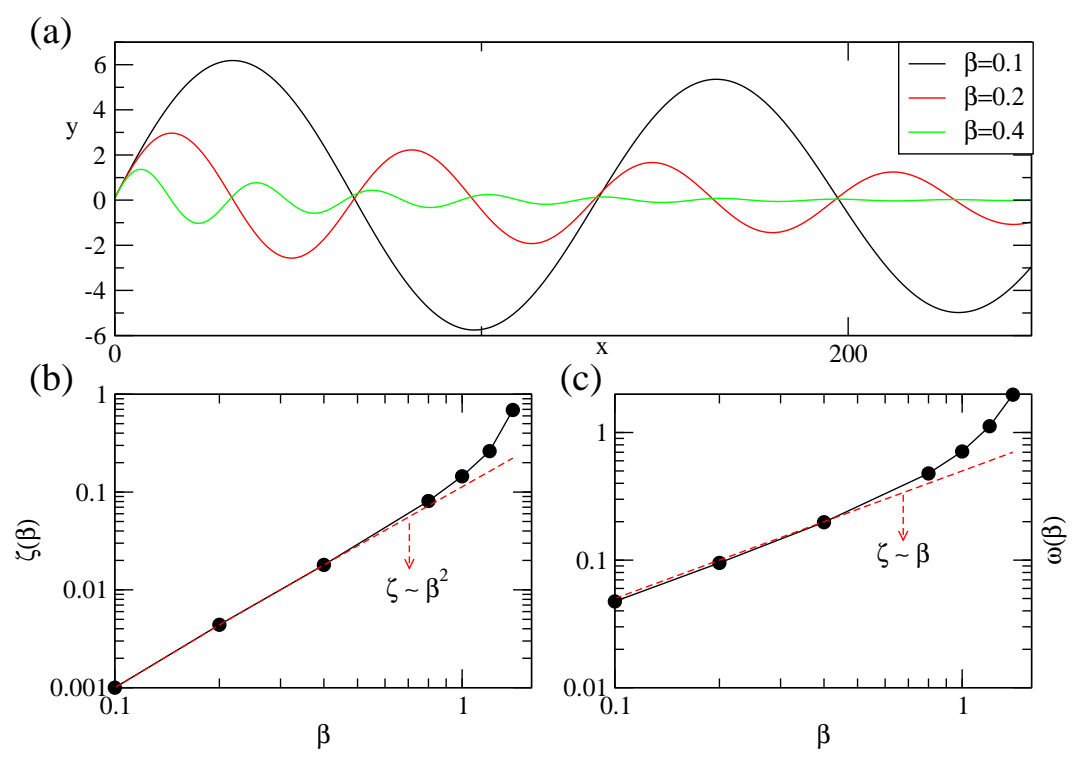

Figure 6: (a): Trajectories of trail-following ants with two-point sensing (2.13), for sensing half-angles $\pi / 10$ (black), $\pi / 5$ (red), and $2 \pi / 5$ (green). The pheromone trail is located horizontally at $y=0$. (b) and (c): log-log plot of damping coefficient $\zeta$ and wavenumber $\omega$ as a function of $\beta$ showing the asymptotic behavior for small $\beta$. 


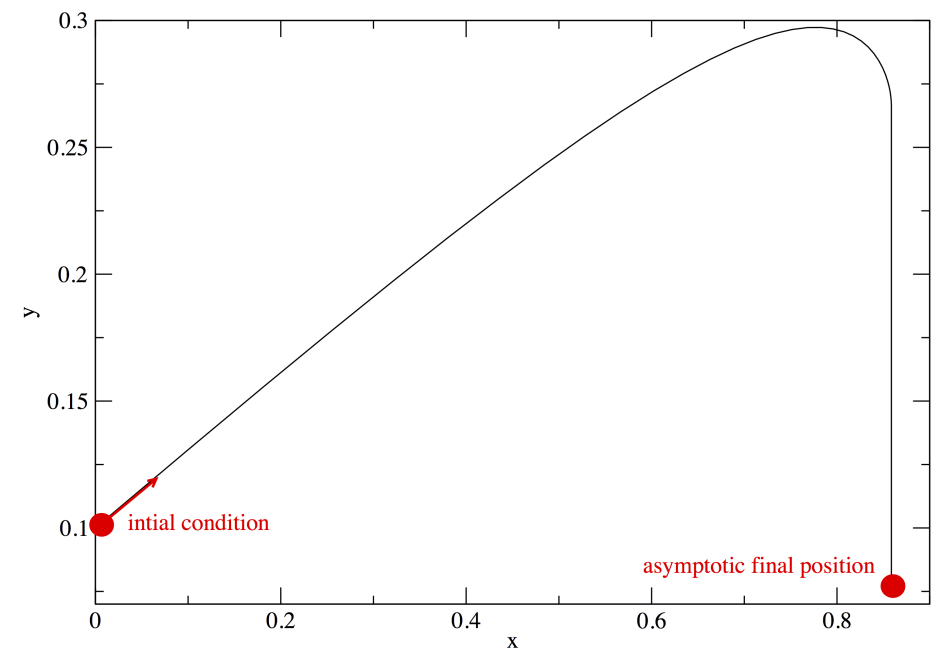

Figure 7: Typical trajectory of an individual ant according to model 2.13), with sensing area larger than a half-circle $(\beta>\pi / 2)$. The pheromone trail is located horizontally at $y=0$. The ant asymptotically approaches a point near the center of the trail and thus no trail following occurs.

\section{Assessment of the model by comparison with experimental results}

In this section, we argue that certain quantities calculated explicitly from our model are in agreement with the experimental results reported in [24]. Of course, our proposed model is, like any other, necessarily incomplete and idealized, and so it remains challenging to assert that agreement with experimental data "validates" the model. Nevertheless, it is possible to indicate that the model is at least consistent with some data. This observation together with the demonstrated robust mathematical properties of the model, suggests that the proposed modeling framework might be a good starting point for further studies of ant navigation.

With this goal in mind, we will consider the turning angle, which we define at each time as the angle between the ant's current velocity $\mathbf{v}$ and its desired velocity $F(\mathbf{x}, \mathbf{v})$. When the pheromone distribution around the ant is supposed to be constant on each half of the sensing area, we are able to explicitly calculate this value.

\subsection{Turning angle distribution}

Following [24], we will suppose that pheromone around an ant is constant on each half of the sensing area $B$, and deduce expressions for the turning angle, 
for both models 2.7$)$ and $(2.13)$.

Let us first consider the model (2.1), 2.7). We denote by $B_{L}$ and $B_{R}$ the circular sectors

$$
\begin{aligned}
& B_{L}(\mathbf{v}, \ell, \beta)=\left\{\mathbf{y} \in \mathbb{R}^{2}: \angle(\mathbf{v}, \mathbf{y}) \in(0, \beta),\|\mathbf{y}\| \leq \ell\right\}, \\
& B_{R}(\mathbf{v}, \ell, \beta)=\left\{\mathbf{y} \in \mathbb{R}^{2}: \angle(\mathbf{v}, \mathbf{y}) \in(-\beta, 0),\|\mathbf{y}\| \leq \ell\right\},
\end{aligned}
$$

so that $B=B_{L} \cup B_{R}$ (see (2.2)). Suppose that the effective pheromone distribution $\mathcal{P}_{e}$ in the desired velocity (2.7) is given by (see Fig. 8)

$$
\mathcal{P}_{e}=c_{L} \mathbb{1}_{B_{L}}+c_{R} \mathbb{1}_{B_{R}} .
$$

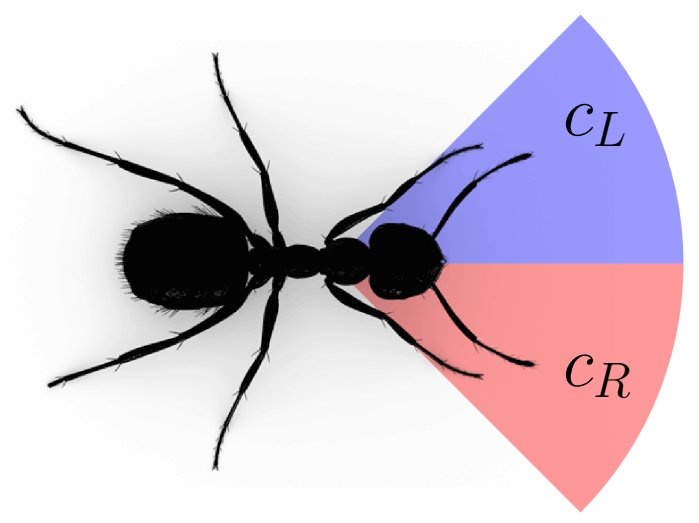

Figure 8: Pheromone distribution used to compute the turning angles.

Then, we compute

$$
F(\mathbf{x}, \mathbf{v})=\lambda \frac{c_{L} \int_{B_{L}} \mathbf{y} d \mathbf{y}+c_{R} \int_{B_{R}} \mathbf{y} d \mathbf{y}}{c_{L}\left|B_{L}\right|+c_{R}\left|B_{R}\right|} .
$$

Using polar coordinates, we find

$$
\begin{aligned}
\left(\int_{B_{L}} \mathbf{y} d \mathbf{y}\right)_{1} & =\int_{0}^{\ell} \int_{\Theta}^{\Theta+\beta} r^{2} \cos \theta d \theta d r \\
& =\frac{\ell^{3}}{3}(\sin (\Theta+\beta)-\sin \Theta), \\
\left(\int_{B_{L}} \mathbf{y} d \mathbf{y}\right)_{2} & =\int_{0}^{\ell} \int_{\Theta}^{\Theta+\beta} r^{2} \sin \theta d \theta d r \\
& =\frac{\ell^{3}}{3}(-\cos (\Theta+\beta)+\cos \Theta),
\end{aligned}
$$


and

$$
\begin{aligned}
\left(\int_{B_{R}} \mathbf{y} d \mathbf{y}\right)_{1} & =\int_{0}^{\ell} \int_{\Theta-\beta}^{\Theta} r^{2} \cos \theta d \theta d r \\
& =\frac{\ell^{3}}{3}(\sin \Theta-\sin (\Theta-\beta)), \\
\left(\int_{B_{R}} \mathbf{y} d \mathbf{y}\right)_{2} & =\int_{0}^{\ell} \int_{\Theta-\beta}^{\Theta} r^{2} \sin \theta d \theta d r \\
& =\frac{\ell^{3}}{3}(-\cos \Theta+\cos (\Theta-\beta)) .
\end{aligned}
$$

After some routine calculations, this gives

$$
\begin{aligned}
c_{L} & \int_{B_{L}} \mathbf{y} d \mathbf{y}+c_{R} \int_{B_{R}} \mathbf{y} d \mathbf{y} \\
& =\frac{\ell^{3}}{3}\left(\left(c_{R}+c_{L}\right) \sin \beta(\cos \Theta, \sin \Theta)+\left(c_{R}-c_{L}\right)(1-\cos \beta)(\sin \Theta,-\cos \Theta)\right) \\
& =\frac{\ell^{3}}{3}\left(\left(c_{R}+c_{L}\right) \sin \beta \frac{\mathbf{v}}{\|\mathbf{v}\|}+\left(c_{R}-c_{L}\right)(1-\cos \beta) \frac{\mathbf{v}^{\perp}}{\|\mathbf{v}\|}\right) .
\end{aligned}
$$

Therefore, the desired velocity $F(\mathbf{x}, \mathbf{v})$ is a multiple of the vector

$$
\left(c_{R}+c_{L}\right) \sin \beta \frac{\mathbf{v}}{\|\mathbf{v}\|}+\left(c_{R}-c_{L}\right)(1-\cos \beta) \frac{\mathbf{v}^{\perp}}{\|\mathbf{v}\|} .
$$

Now, we define the turning angle $\alpha_{1}=\alpha_{1}(\beta)$ as the angle between $\mathbf{v}$ and $F$. Thus,

$$
\alpha_{1}(\beta)=\arctan \left(\frac{\left(c_{R}-c_{L}\right)(1-\cos \beta)}{\left(c_{R}+c_{L}\right) \sin \beta}\right)=\arctan \left(\frac{c_{R}-c_{L}}{c_{R}+c_{L}} \tan \frac{\beta}{2}\right) .
$$

Proceeding in a similar way with the model with pointwise sensing 2.1$], 2.13$, we find for the turning angle $\alpha_{2}(\beta)$

$$
\alpha_{2}(\beta)=\arctan \left(\frac{c_{R}-c_{L}}{c_{R}+c_{L}} \tan \beta\right)=\alpha_{1}(2 \beta) .
$$

Observing the relation between the two turning angles, we can see that for the purposes of calculating the turning angle, the model 2.7) with sensing on the whole sensing area is equivalent to the model 2.13 with sensing points situated at an angle of $\beta / 4$ around the ant's direction.

\subsection{Consistency of the model with the turning angle dis- tribution in the Argentine ant}

We can use the expressions $(5.1),(5.2)$ for the turning angles to check if they are consistent with the experimental results of [24] for the Argentine ants (Linepithema humile). In that work, the angle of the sensing area is not a parameter 
of the model. Therefore one test of our model is to determine which sensing area half-angle $\beta$ best approximates the turning angle distributions reported in [24], and if such a value for the angle is reasonable or supported by other considerations.

In [24, Fig. 5], the authors report the results of experiments measuring the turning angle of the Argentine ant in response to differences in pheromone distribution on both sides of the ant. Specifically (using our notations), they plot for various values of total pheromone $c_{L}+c_{R}$ the turning angle as a function of the pheromone difference $c_{R}-c_{L}$, and compute the slope $k$ of that graph at the origin. In our model, since for fixed $c_{L}+c_{R}$ and $\beta$ the turning angle is given by (5.1) or 5.2), the corresponding slopes $k_{1}, k_{2}$ will be

$$
k_{1}=\frac{\tan \frac{\beta}{2}}{c_{R}+c_{L}}, \quad k_{2}=\frac{\tan \beta}{c_{R}+c_{L}} .
$$

In Table 1, we present the values of $k_{2}$ for different values of total pheromone $c_{L}+c_{R}$, and different sensing half-angles $\beta$. The data (along with the same data for $\beta=\pi / 10$ ) is shown in Table 1 and Figure 9. We can see that the slope is best approximated if we take a sensing half-angle close to $\beta=2 \pi / 13$.

The fact that we obtain a particular value for $\beta$ indicates that there may be some relevant selection criteria at work for the angle. This suggests that it would be interesting to test (either experimentally or numerically) how varying the sensing angle impacts on the ant's navigation efficiency.

\begin{tabular}{|l|c|c|c|c|c|}
\hline$c_{R}+c_{L}$ & $\beta=\pi / 4$ & $\pi / 5$ & $2 \pi / 13$ & $\pi / 7$ & {$[24$, Fig. 5$]$} \\
\hline \hline 57 & 1.005 & 0.73 & 0.528 & 0.484 & 0.498 \\
\hline 115 & 0.498 & 0.362 & 0.261 & 0.24 & 0.282 \\
\hline 229 & 0.25 & 0.182 & 0.131 & 0.12 & 0.142 \\
\hline 459 & 0.125 & 0.0906 & 0.0655 & 0.0601 & 0.066 \\
\hline 918 & 0.0624 & 0.0453 & 0.0328 & 0.0301 & 0.031 \\
\hline 1836 & 0.0312 & 0.0227 & 0.0164 & 0.015 & 0.015 \\
\hline
\end{tabular}

Table 1: The slope $k_{2}$ corresponding to the model 2.13, for different sensing half-angles $\beta$, as a function of the total pheromone $c_{L}+c_{R}$. For each line, we chose $c_{L}+c_{R}$ as the midpoint of the range in each of the plots in [24, Fig. 5].

Let us further see if the value $\beta=2 \pi / 13$ is consistent with the data obtained in 24] relating to the sensing area. To achieve this, consider [24, Fig. 7]. There, the authors show a heat map of what, according to their reasoning, can be interpreted as the area where the presence of pheromone around the ant correlates better with changes in direction. Thus this area can be interpreted as a sensing area.

In Fig. 10, we have superimposed on [24, Fig. 7] the line segments originating from the a point slightly behind the center of the ant (to account for the perception delay), using the angle $2 \pi / 13$ found above. By choosing this point in such a way that the line segments align better with the correlation heat map, 


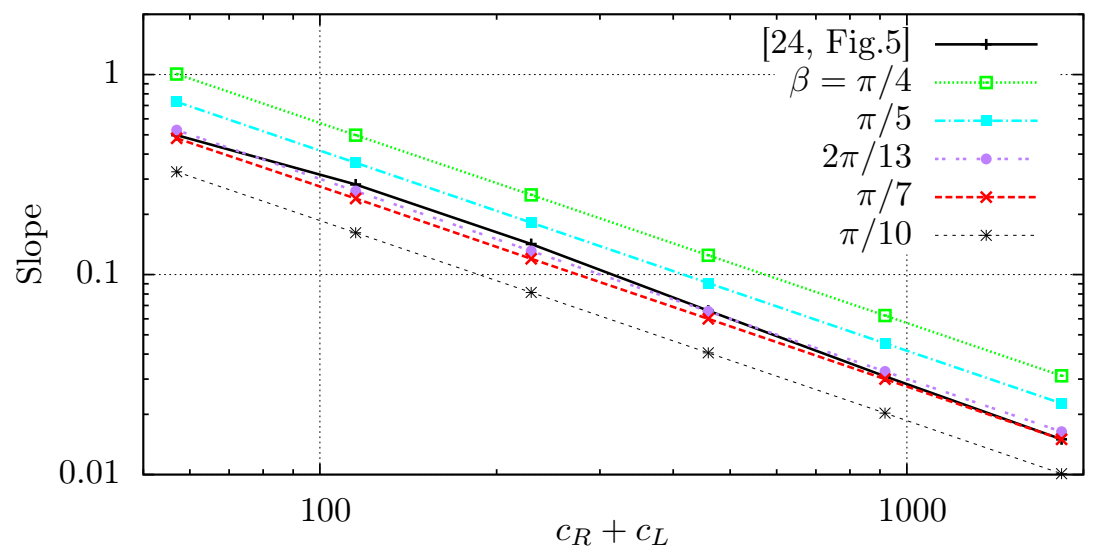

Figure 9: Log plot of the data in Table 1.

and knowing the typical speed of the ant at $2 \mathrm{~cm} / \mathrm{sec}$, we obtain a reaction delay time of approximately 0.2 seconds. This value is supported by the observation in 24] that their data are consistent with a small perception delay. Additionally, we note that the fact that (allowing for the small perception delay) the red segments in Figure 10 can be made to align reasonably well with the correlation map from [24] indicates that the results from our model fall into biologically realistic ranges.

\section{Conclusions and outlook}

We have introduced and analyzed an individual-based model specifically tailored for ant navigation. The main aspects are a generalization of Weber's Law using a sensing area around the ant representing the pheromone detection area. We have shown that the model exhibits good mathematical properties, especially when the sensing area has a span of less than $\pi$; that is, when ants do not detect pheromone situated behind them. In that case, we proved that the ant's speed will tend to a range of natural velocities which are bounded and bounded away from zero. This result is not true if the sensing area reaches behind the ant.

We proved, under reasonable assumptions - include a sensing area smaller than a half-circle - that pheromone-trail-following solutions exhibit stability properties. These properties even extend, in a weaker form, to trails with variations in pheromone distribution along their length. For homogeneous trails, linear stability of the corresponding trail-following solution is obtained, and a Lyapunov function is found.

The question arises of whether trail following happens only when the sensing area is smaller than a half-circle, and, more generally, in what way does the value of the angle affect trajectory shape. We address this question with numerical 


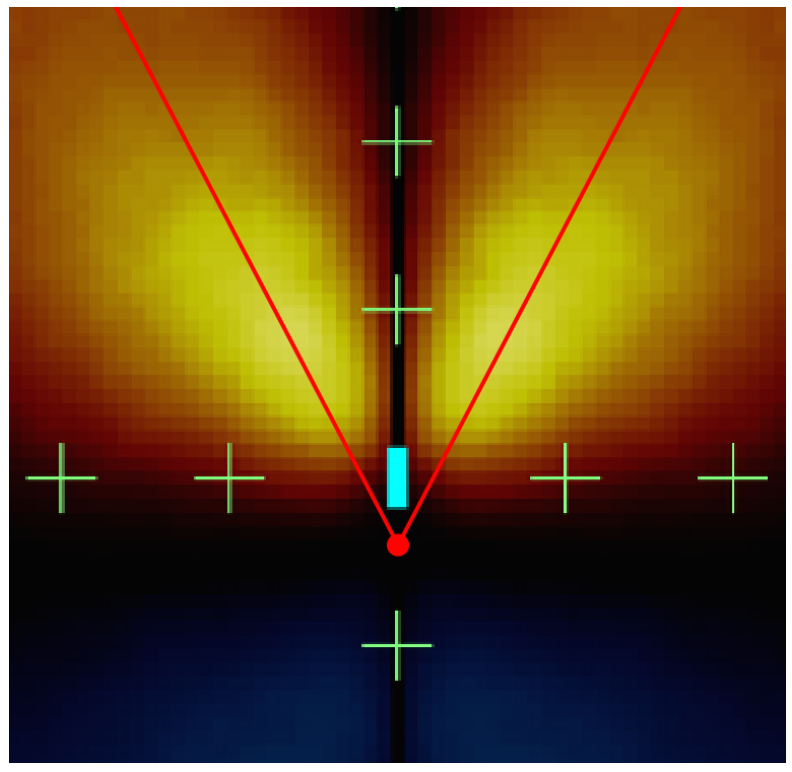

Figure 10: Antennal span superimposed on Fig.5 from 24] (adapted with permission). The blue segment represents the ant, and each green cross is $1 \mathrm{~cm}$ apart.

experiments showing that the trail following trajectories behave like overdamped oscillations around the trail, and that for sensing areas larger than a half-circle, the ant is not able to follow a trail.

To assess the consistency of our model with previous experimental results, we used it to calculate the turning angle distribution and found it to be in agreement with the values found experimentally in [24].

After having studied how individuals ants interact with a pheromone landscape, the next natural step would be to include pheromone production and explore the potential collective effects that emerge among interacting, pheromone producing ants. The obtained collective behavior could then be compared with the results obtained in, for instance, [4. It would be interesting to study the spontaneous emergence of pheromone trails and investigate their properties as well as the emergence of collective behaviors, in the spirit of [8, 9, 12]. Then, it would be appealing to discuss hierarchies of models, and to investigate whether hydrodynamic descriptions could be relevant, as in [7, 10, 18, 2]. We intend to address these questions in further work.

\section{Acknowledgements}

We acknowledge support form the Brazilian-French Network in Mathematics, which has made possible a visit in Nice where a large part of this work was done. 
P.A. was partially supported by FAPERJ "Jovem Cientista do Nosso Estado" grant no. 202.867/2015, and CNPq grant no. 442960/2014-0.

\section{References}

[1] E. B. Bandeira de Melo and A. F. R. Araújo. Modelling foraging ants in a dynamic and confined environment. BioSystems, 104:23-31, 2011.

[2] L. Barberis and F. Peruani. Large-scale patterns in a minimal cognitive flocking model: Incidental leaders, nematic patterns, and aggregates. Phys. Rev. Lett., 117:248001-248006, 2016.

[3] R. Beckers, J.-L. Deneubourg, and S. Goss. Trail laying behaviour during food recruitment in the ant lasius niger (L.). Insectes soc., 39(1):59-72, 1992.

[4] E. Boissard, P. Degond, and S. Motsch. Trail formation based on directed pheromone deposition. J. Math. Biol., 66(6):1267-1301, 2013.

[5] V. Calenbuhr and J.-L. Deneubourg. A model for osmotropotactic orientation (I). J. Theor. Biol., 158(3):359-393, 1992.

[6] V. Calenbuhr and J.-L. Deneubourg. A model for osmotropotactic orientation (II). J. Theor. Biol., 158(3):395-407, 1992.

[7] J. A. Carrillo, M. Fornasier, G. Toscani, and F. Vecil. Particle, kinetic, and hydrodynamic models of swarming, pages 297-336. Modeling and Simulation in Science, Engineering and Technology. Birkhauser, 2010.

[8] F. Cucker and S. Smale. Emergent behavior in flocks. IEEE Trans. Automat. Control, 52:852-862, 2007.

[9] F. Cucker and S. Smale. On the mathematics of emergence. Japan. J. Math., 2:197-227, 2007.

[10] P. Degond, A. Frouvelle, and J.-G. Liu. Macroscopic limits and phase transition in a system of self-propelled particles. J. Nonlinear Sc., 23(3):427456, 2013.

[11] J.-L. Deneubourg, S. Aron, S. Goss., and J.M. Pasteels. The self-organizing exploratory pattern of the Argentine ant. J. Insect Behavior, 3(2):150-168, 1990.

[12] M. R. D’Orsogna, Y. L. Chuang, A. L. Bertozzi, and L. S. Chayes. Selfpropelled particles with soft-core interactions: Patterns, stability, and collapse. Phys. Rev. Lett., 96(10):104302, 2006.

[13] L. Edelstein-Keshet. Simple models for trail-following behaviour; trunk trails versus individual foragers. J. Math. Biol., 32:303-328, 1994. 
[14] L. Edelstein-Keshet, J. Watmough, and B.G. Ermentrout. Trail following in ants: individual properties determine population behaviour. Behavioral Ecology and Sociobiology, 36(2):119-133, 1995.

[15] B. Ehmer and W. Gronenberg. Antennal muscles and fast antennal movements in ants. J. Comparative Physiology B, 167(4):287-296, 1997.

[16] M. A. Fontelos, A. Garnier, and M. Vela-Perez. From individual to collective dynamics in Argentine ants (linepithema humile). Mathematical Biosciences, 262:56-64, 2015.

[17] M.A. Fontelos and A. Friedman. A PDE model for the dynamics of trail formation by ants. J. Math. Anal. Appl., 425(1):1-19, 2015.

[18] S.-Y. Ha and E. Tadmor. From particle to kinetic and hydrodynamic descriptions of flocking. Kinet. Relat. Models, 1(3):415-435, 2008.

[19] W. Hangartner. Spezifität und inaktivierung des spurpheromons von lasius fuliginosus latr. und orientierung der arbeiterinnen im duftfeld. Zeitschrift für vergleichende Physiologie, 57(2):103-136, 1967.

[20] B. Hölldobler and E.O. Wilson. The Ants. The Belknap Press of Harvard University Press, 1990.

[21] D. Jackson, M. Holcombe, and F. Ratnieks. Coupled computational simulation and empirical research into the foraging system of Pharaoh's ant (monomorium pharaonis). Biosystems, 76(1-3):101-112, 2004.

[22] A. John, A. Schadschneider, D. Chowdhury, and K. Nishinari. Traffic-like collective movement of ants on trails: absence of jammed phase. Phys. Rev. Lett., 102:108001, 2009.

[23] K. Johnson and L. F. Rossi. A mathematical and experimental study of ant foraging trail dynamics. J. Theor. Biol., 241:360-369, 2006.

[24] A. Perna, B. Granovskiy, S Garnier, Nicolis S.C., M. Labédan, G. Theraulaz, V. Fourcassié, and D. J. T. Sumpter. Individual rules for trail pattern formation in Argentine ants (linepithema humile). PLoS Comput Biol., 8(7):e1002592, 2012.

[25] K. Ramsch, Reid C. R., M. Beekman, and M. Middendorf. A mathematical model of foraging in a dynamic environment by trail-laying Argentine ants. J. Theor. Biol., 306:32-45, 2012.

[26] C. R. Reid, T. Lattya, and M. Beekam. Making a trail: informed Argentine ants lead colony to the best food by U-turning coupled with enhanced pheromone laying. Animal Behaviour, 84(6):1579-1587, 2012.

[27] S. D. Ryan. A model for collective dynamics in ant raids. J. Math. Biol., 72:1579-1606, 2015. 
[28] Camazine S., Deneubourg J.-L., Franks N. R., J. Sneyd, G. Theraulaz, and E. Bonabeau. Self-organization in biological systems. Princeton studies in complexity. Princeton University Press, 2001.

[29] F. Schweitzer, K. Lao, and F. Family. Active random walkers simulate trunk trail formation by ants. BioSystems, 41:153-166, 1997.

[30] T. Sharpe and B. Webb. Simulated and situated models of chemical trail following in ants. In Proc. 5th Int. Conf. Simulation of Adaptive Behavior, pages 195-204. MIT Press, 1998.

[31] T. Vicsek and A. Zafeiris. Collective motion. Phys. Reports, 517(3-4):71$140,2012$.

[32] J. Watmough and L. Edelstein-Keshet. A one-dimensional model of trail propagation by army ants. J. Math. Biology, 33:459-476, 1995.

[33] E. O. Wilson. Chemical communication among workers of the fire ant solenopsis saevissima (Fr. Smith). 1. The organization of mass-foraging. Animal Behaviour, 10(1-2):134-138, 1962.

[34] E. O. Wilson. Chemical communication among workers of the fire ant solenopsis saevissima (Fr. Smith). 2. An information analysis of the odour trail. Animal Behaviour, 10(1-2):148-158, 1962. 